Universidade de São Paulo

Instituto de Física

\title{
Descrição pseudoclássica de férmions quirais que interagem com campo de matéria e com o campo eletromagnético
}

\author{
Agnes Ferreira de Souza
}

Orientador: Prof. Dr. Dmitri Maximovitch Guitman

Dissertação de mestrado apresentada ao Instituto de Física da Universidade de São Paulo, como requisito parcial para a obtenção do título de Mestre em Ciências.

Banca Examinadora:

Prof. Dr. Dmitri Maximovitch Guitman (IFUSP)

Prof. Dr. Dmitry Vasilevich (UFABC)

Prof. Dr. Jeferson de Lima Tomazelli (UFSC)

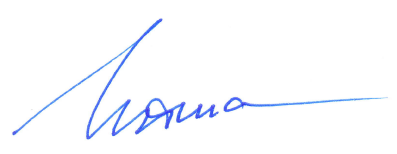

São Paulo 
FICHA CATALOGRÁFICA

Preparada pelo Serviço de Biblioteca e Informação do Instituto de Física da Universidade de São Paulo

\section{Souza, Agnes Ferreira}

Descrição pseudoclássica de férmions quirais que interagem com campo de matéria e com o campo eletromagnético. São Paulo, 2021.

Dissertação (Mestrado) - Universidade de São Paulo, Instituto de Física, Depto. de Física Nuclear.

Orientador: Prof. Dr. Dmitri Maximovitch Guitman

Área de Concentração: Física

Unitermos: 1. Teoria quântica relativística; 2. Física teórica; 3. Mecânica quântica.

USP/IF/SBI-021/2021 
University of São Paulo

Physics Institute

\title{
Pseudoclassical description of chiral fermions interacting with matter fields and with eletromagnetic fields
}

\author{
Agnes Ferreira de Souza
}

Supervisor: Prof. Dr. Dmitri Maximovitch Guitman

Dissertation submitted to the Physics Institute of the University of São Paulo in partial fulfillment of the requirements for the degree of Master of Science.

Examining Committee:

Prof. Dr. Dmitri Maximovitch Guitman (IFUSP)

Prof. Dr. Dmitry Vasilevich (UFABC)

Prof. Dr. Jeferson de Lima Tomazelli (UFSC) 



\section{Agradecimentos}

Agradeço ao meu orientador, Prof. Dr.Dmitri Maximovitch Guitman pela orientação e ao Dr. João Luis Meloni Assirati e ao Prof. Dr. Rodrigo Fresneda pela ajuda e discussões.

O presente trabalho foi realizado com apoio do CNPq, Conselho Nacional de Desenvolvimento Científico e Tecnológico - Brasil. Agradeço ao CNPq pelo apoio financeiro. 


\section{Resumo}

O efeito magnético quiral é um efeito quântico macroscópico em que se observa a geração de uma corrente elétrica induzida pelo desequilíbrio da quiralidade de férmions na presença de um campo magnético externo. Este efeito já foi observado experimentalmente e tem atraído grande interesse. Sua teoria e aplicações estão em fase de desenvolvimento.

Neste trabalho construímos um modelo pseudoclássico de um sistema de interesse para o estudo do efeito magnético quiral, composto por férmions quirais massivos que interagem com um campo eletromagnético externo e com um campo de matéria através de interação eletrofraca. Para isso, o sistema é descrito por uma equação de Dirac modificada e é aplicado o método de representação por integrais de trajetória de uma função de Green desenvolvido por E. S. Fradkin e D. M. Gitman [1]. Para comprovar a validade do modelo obtido, verificamos que a quantização de Dirac do modelo recupera o sistema inicial.

Além do modelo pseudoclássico, foi possível também obter outros resultados inéditos para esse sistema, como a representação da função de Green causal por integrais de trajetória (formulação de Feynmann da mecânica quântica) e a teoria Hamiltoniana com vínculos do sistema.

Palavras-chaves: Efeito magnético quiral, modelo pseudoclássico, integrais de trajetória. 


\section{Abstract}

The chiral magnetic effect is a macroscopic quantum phenomenon in which the generation of an induced electric current is observed due to the imbalance of the chirality of fermions associated with the presence of an external magnetic field. This effect has been observed experimentally and has been attracting great interest. Its theory and applications are still currently in development by the scientific community.

In this work we built a pseudoclassical model of a system of interest to the study of the chiral magnetic effect, composed of massive chiral fermions that interact with an electromagnetic field and with a matter field through electroweak interaction. For this study, the system is described by a modified Dirac equation and the method of representation by path integrals of a Green function developed by E. S. Fradkin and D. M. Gitman [1] is applied. To prove the validity of the model, we verified that the Dirac quantization recovers the original system.

Besides the pseudoclassical model, other unprecedented results were possible to obtain about this system, such as the representation of the causal Green function by path integrals (Feynmann's formulation of quantum mechanics) and the Hamiltonian theory with constraints to the system.

Keywords: Chiral magnetic effect, pseudoclassical model, path integral. 


\section{Sumário}

\begin{tabular}{lll}
\hline 1 & Introdução & 1
\end{tabular}

2 Modelo clássico de uma partícula de Klein- Gordon que interage com o campo eletromagnético 5

2.1 Ação clássica . . . . . . . . . . . . . . . . . . 5

3 Modelo pseudoclássico de uma partícula de Dirac que interage com o campo $\begin{array}{ll}\text { eletromagnético } & 11\end{array}$

3.1 Ação pseudoclássica . . . . . . . . . . . . . . . . . . . . 12

4 Modelo pseudoclássico de uma partícula de Dirac que interage com o campo eletromagnético e interage, através da interação eletrofraca, com o campo de matéria 21

4.1 Ação pseudoclássica . . . . . . . . . . . . . . . . . . . . . 22

4.2 Quantização da ação pseudoclássica. . . . . . . . . . . . . . . 28

$\begin{array}{lll}5 & \text { Conclusão } & 36\end{array}$

$\begin{array}{lll}6 & \text { Apêndice } & 38\end{array}$ 


\section{CAPÍTULO 1}

\section{Introdução}

A construção de modelos pseudoclássicos começou em 1975, quando F. A. Berezin e M. S. Marinov, com o objetivo de encontrar uma maneira de formular a mecânica Hamiltoniana para sistemas que possuem spin, investigaram o uso da álgebra de Grassmann. Os elementos desta álgebra anticomutam, com eles formamos variáveis que comutam (variáveis de Grassmann pares) e variáveis que anticomutam (variáveis de Grassmann ímpares). As variáveis pares e ímpares de Grassmann são elementos de uma outra álgebra, formulada posteriormente por F. A. Berezin e chamada de álgebra de Berezin. Usamos essa álgebra para descrever sistemas com spin semi-inteiro [2].

Ao encontrarmos a ação de um sistema temos o seu modelo clássico, no entanto, quando obtemos a ação de um sistema escrito com as variáveis de Grassmann, esse modelo é chamado de pseudoclássico.

No ano seguinte, Berezin e Marinov desenvolveram essa teoria e a aplicaram em alguns sistemas [3]. Obtiveram a ação do sistema composto por uma partícula não relativística com spin $1 / 2$ e a quantizaram, a quantização forneceu a eles a teoria usual de uma partícula não relativística com spin $1 / 2$, confirmando a validade da ação obtida. O mesmo foi feito com o sistema descrito pela partícula relativística com spin 1/2. Outros autores também investigaram este último sistema [1, 3, 4, 5, 6, 7, 8, 9, 10, 11].

Outros sistemas foram propostos, como a generalização da ação pseudoclássica de uma partícula com spin semi-inteiro e com momento magnético anômalo, desenvolvida na Ref. [12], e uma versão não comutativa da ação de Berezin-Marinov, que foi construída na Ref.[13].

Na referência [14] é apresentado um modelo pseudoclássico cuja quantização reproduz a teoria das partículas de Weyl; além disso, neste mesmo trabalho, foi analisado também um modelo pseudoclássico para partículas relativísticas com spin 1, no caso de uma ação com o termo de Chern-Simons e para os casos com e sem massa. Ao final, esta referência mostra que a mecânica quântica construída para o caso massivo é equivalente à teoria da Proca e à teoria de Maxweel no caso sem massa.

O modelo pseudoclássico para uma partícula de Dirac massiva em $2+1$ dimensões foi construído na Ref.[15] e generalizado para os casos de dimensão par e ímpar nas 
Ref.[16, 17, 18, 19]. Ações pseudoclássicas de partículas com spin semi-inteiro em um background não-abeliano e em um campo de torção foram propostas nas Refs. [20] e [21], respectivamente.

Nos trabalhos citados acima é possível observar diferentes formas de se obter e quantizar modelos pseudoclássicos. Dentre elas esta o método de E. S. Fradkin e D. M. Gitman, usado neste trabalho, onde a função de Green causal do sistema é representada através de integrais de trajetórias, as integrais também são feitas ao longo das trajetórias associadas com as variáveis de Grassmann [1]. Ao final do processo é obtido o modelo pseudoclássico e a sua representação por integrais de trajetória. As representações por integrais de trajetória são frequentemente um método eficiente para o cálculo de propagadores, veja por exemplo [22, 23]. Elas também fundamentam o chamado método da linha de universo na teoria das cordas e na teoria quântica de campos, veja por exemplo [24].

Outra linha de pesquisa relacionada a esta, é a construção de modelos de partículas relativísticas, ainda representadas por integrais de trajetória, porém escritas exclusivamente com variáveis bosônicas. Estes modelos são capazes de descrever efeitos físicos mais claramente, no entanto, a sua estrutura, como regra, é tecnicamente mais complicada que a estrutura dos modelos pseudoclássicos.

O trabalho [25] mostrou que é possível obter uma quantização que reproduz a mecânica quântica de partículas com spin semi-inteiro por meio desses modelos que usam variáveis bosônicas. E, recentemente, a referência [26] mostrou que trajetórias bosônicas de um modelo clássico que descreve férmions quirais sem massa em um campo magnético externo podem ser usados para calcular o efeito magnético quiral.

O EMQ é um efeito quântico macroscópico que ocorre em sistemas que possuem férmions quirais carregados e que interagem com o campo magnético. Ao expormos estas partículas a um campo magnético ocorre uma quebra da invariância por rotação destes férmions, criando uma orientação preferencial para seus spins semi-inteiros, o que gera uma diferença no número de partículas de mão-direita e de mão-esquerda do sistema, induzindo a geração de uma corrente elétrica anômala na direção do campo magnético.

A primeira descrição teórica do EMQ foi feita, aparentemente, por A. Vilenkin em 1980 [27]. Neste artigo foi estudada a interação entre partículas de mão-esquerda carregadas, sem massa, e o campo magnético; foi obtida uma corrente de partículas carregadas na direção do campo magnético. Outro sistema estudado neste trabalho foi o dos férmions massivos e carregados que interagem por meio da força eletrofraca. No entanto, na ordem mais baixa da teoria de perturbação, a análise realizada indicou a ausência de uma corrente anômala gerada no sistema.

Nos anos de 1978 e 1979, Vilenkin já havia obtido, teoricamente, a geração de uma corrente anômala para o caso de partículas sem massa e de mão-esquerda, mas, di- 
ferentemente do trabalho de 1980, elas estavam em rotação, o que gerou uma corrente paralela ao vetor da velocidade angular [28, 29]. Esses trabalhos despertaram um grande interesse no estudo do efeito magnético quiral - veja, por exemplo, as refs. [30, 31, 32, 33, 34, 35, 36, 37, 38, 39, 40].

Uma revisão histórica mais detalhada do EMQ pode ser encontrada na ref. [41], na qual destaca-se a observação deste efeito nos semimetais de Weyl [42, 43, 44, 45, 46, 47, 48] e a relação do efeito com a anomalia de Adler-Bell-Jackiw [51, 52].

$\mathrm{O}$ efeito magnético quiral foi observado experimentalmente, pela primeira vez, no semimetal de Dirac $Z r T e_{5}$, por meio do experimento [49]. Os autores mostraram que a observação do efeito se torna possível devido a uma transformação efetiva do semimetal de Dirac em um semimetal de Weyl quando exposto a campos magnéticos e elétricos paralelos. Existem também hipóteses sobre a ocorrência deste efeito na cosmologia, a saber, nos processos associados à supernovas [50], e nos processos de colisões de íons pesados [53].

Além disso, o trabalho [26] também estudou o comportamento semiclássico de líquidos de Fermi próximo à superfície de Fermi [54]. A análise deles é baseada na ação clássica de partículas de Weyl carregadas e de spin $\frac{1}{2}$. Um dos resultados obtidos é o de que as trajetórias da ação clássica do sistema podem ser usadas para calcular a corrente de férmions carregados, já que elas fornecem o valor exato da corrente gerada. Este é mais um exemplo da importância do estudo de modelos clássicos e pseudoclássicos.

A referência [55] obtêm as soluções exatas de uma série de equações de Dirac que incluem o potencial efetivo responsável pela interação das partículas com o campo de matéria. Para estudarmos o efeito magnético quiral usamos a seguinte equação retirada do artigo [56]:

$$
\left[\left(i \partial_{\mu}-g A_{\mu}(x)\right) \gamma^{\mu}-m-\gamma_{\mu}\left(V_{L}^{\mu}(x) P_{L}+V_{R}^{\mu}(x) P_{R}\right)\right] \Psi(x)=0,
$$

onde $V_{L, R}^{\mu}(x)$ são os potenciais efetivos da interação eletrofraca entre o campo de matéria e os elétrons de mão direita e de mão esquerda respectivamente. $P_{L, R}=\left(1 \mp \gamma^{5}\right) / 2$ são as projeções quirais, e $\gamma^{\mu} \operatorname{com} \mu=0,1,2,3$ e $\gamma^{5}=i \gamma^{0} \gamma^{1} \gamma^{2} \gamma^{3}$ são as matrizes gamma de Dirac.

A equação de onda relativística acima descreve férmions quirais que interagem com o campo de matéria, através da força eletrofraca, e com o campo eletromagnético. A referência [56], estudou o comportamento das partículas quirais expostas ao campo magnético, e se podemos esperar, no limite quiral, o aparecimento de correntes anômalas devido à interação com o campo da matéria. O estudo da geração desta corrente pode ser feito de modo similar ao feito por Vilenkin [27], onde foi feita a análise das soluções da equação de Dirac modificada, ou como foi feito no trabalho [26], onde foi usado a ação clássica. No entanto, neste trabalho vamos focar na construção do modelo 
pseudoclássico e na sua quantização.

\section{Divisão do trabalho}

No capítulo 1, introdução, foi feita uma revisão de alguns dos modelos pseudoclássicos já desenvolvidos e do Efeito Magnético Quiral.

Nos capítulos 2 e 3 estudaremos o artigo de Fradkin e Gitman que inaugura o método aqui usado para obter modelos pseudoclássicos [1]. As contas deste artigo são refeitas com o objetivo pedagógico de compreender o método. Vale lembrar que este método parte da equação quântica do sistema e ao final do processo obtemos a sua função de Green causal escrita como uma integral de trajetória.

No capítulo 4 aplicamos o método aprendido nos capítulos anteriores em um novo sistema: o sistema de férmions quirais que interagem com o campo de matéria, o que nos fornece o seu modelo pseudoclássico. Na segunda parte do capítulo 4 quantizamos o modelo pseudoclássico obtido, com o objetivo de verificar se o resultado da quantização de Dirac nos fornece a equação quântica com que iniciamos o processo de construção do modelo pseudoclássico do sistema, usamos a referência [57]. E por fim, no capítulo 5, apresentamos a conclusão do trabalho.

Nos próximos parágrafos detalho os modelos estudados em cada um dos capítulos.

No capítulo 2 refazemos as contas do artigo [1] que constroem o modelo clássico de uma partícula escalar relativística que interage com um campo eletromagnético externo e é descrita pela equação de Klein-Gordon modificada para incluir o potencial eletromagnético:

$$
\left[\left(i \partial_{\mu}-g A_{\mu}(x)\right)^{2}-m^{2}\right] \Psi(x)=0 .
$$

No capítulo 3 também refazemos as contas do artigo [1] que constroem o modelo pseudoclássico de uma partícula com spin semi-inteiro, massiva, que interage com o campo eletromagnético externo e é descrita pela equação de Dirac modificada para incluir o potencial eletromagnético:

$$
\left[\left(i \partial_{\mu}-g A_{\mu}(x)\right) \gamma^{\mu}-m\right] \Psi(x)=0
$$

E no capítulo 4, aplicamos o método apresentado nos capítulos anteriores ao sistema descrito pela eq. 1.1. Este sistema e o sistema do capítulo 3 são descritos por equações de Dirac modificadas, quando aplicamos o método nestes dois sistemas vemos muitas semelhanças nos processos que devem ser feitos, por isso, muitos dos resultados usados no capítulo 3, são usados no capítulo 4 e estão melhor detalhados no capítulo 3 . 


\section{CAPÍTULO 2}

\section{Modelo clássico de uma partícula de Klein- Gordon que interage com o campo eletromagnético}

Neste capítulo iremos reproduzir as contas da referência [1]. Este passo é importante para que possamos ter uma visão geral do método desenvolvido por Fradkin e Gitman. Vamos obter, como na referência estudada, a ação clássica para a seguinte equação de Klein-Gordon modificada:

$$
\left[\left(i \partial_{\mu}-g A_{\mu}(x)\right)^{2}-m^{2}\right] \Psi(x)=0,
$$

onde utilizamos que a métrica $\eta_{\mu v}=\operatorname{diag}(1,-1,-1,-1), A_{\mu}(x)$ é o potencial eletromagnético externo, $\operatorname{com} \mu=0,1,2,3$, e $g$ é a carga elétrica.

Esta equação descreve o sistema composto por uma partícula escalar relativística que interage com um campo eletromagnético externo. Nas contas seguintes usaremos que $|x\rangle$ e $|y\rangle$ são os autovetores dos operadores posição $X^{\mu}$ e $Y^{\mu}$, respectivamente, que $P_{\mu}$ é o operador momento linear e que estes operadores e autovetores satisfazem as seguintes relações:

$$
\begin{aligned}
& X^{\mu}|x\rangle=x^{\mu}|x\rangle,\langle x \mid y\rangle=\delta^{4}(x-y), \int|x\rangle\langle x| d x=I, \\
& {\left[P_{\mu}, X^{v}\right]_{-}=-i \delta_{\mu}^{v}, P_{\mu}|p\rangle=p_{\mu}|p\rangle,\left\langle p \mid p^{\prime}\right\rangle=\delta^{4}\left(p-p^{\prime}\right),} \\
& \int|p\rangle\langle p| d p=I,\left\langle x\left|P_{\mu}\right| y\right\rangle=-i \partial_{\mu} \delta^{4}(x-y),\langle x \mid p\rangle=(2 \pi)^{-2} e^{i p x}, \\
& {\left[\Pi_{\mu}, \Pi_{\nu}\right]_{-}=-i g \mathbb{F}_{\mu \nu}(X), \Pi_{\mu}=-P_{\mu}-g A_{\mu}(X) .}
\end{aligned}
$$

\subsection{Ação clássica}

Para iniciarmos este processo devemos primeiro escrever a eq. (2.1) em função da sua função de Green causal $D^{\mathrm{c}}(x, y)$, que satisfaz a equação abaixo:

$$
\left[\left(i \partial_{\mu}-g A_{\mu}(x)\right)^{2}-m^{2}+i \epsilon\right] D^{\mathrm{c}}(x, y)=-\delta^{4}(x-y),
$$


De acordo com Schwinger [58], podemos escrever a função $D^{\mathrm{c}}(x, y)$ como um elemento de matriz do operador $D^{\mathrm{c}}$,

$$
D^{\mathrm{C}}(x, y)=\left\langle x\left|D^{\mathrm{C}}\right| y\right\rangle \text {. }
$$

Usando a equação acima, temos que $\int|z\rangle\langle z| d^{4} z=I$ e $\langle x \mid y\rangle=\delta^{4}(x-y)$. Assim, a eq. 2.3. pode ser escrita como

$$
\int\left[\left(i \partial_{\mu}-g A_{\mu}(x)\right)^{2}-m^{2}+i \epsilon\right]\langle x \mid z\rangle\left\langle z\left|D^{\mathrm{c}}\right| y\right\rangle d^{4} z=-\langle x \mid y\rangle
$$

Definindo os termos $\left(i \partial_{\mu}-g A_{\mu}(x)\right)$ como o operador $\Pi_{\mu}=-P_{\mu}-g A_{\mu}(X)$, podemos obter que

$$
\begin{aligned}
& \int\left\langle x\left|\left(\Pi_{\mu}^{2}-m^{2}+i \epsilon\right)\right| z\right\rangle\left\langle z\left|D^{\mathrm{c}}\right| y\right\rangle d^{4} z=-\langle x \mid y\rangle, \\
& \left\langle x\left|\left(\Pi_{\mu}^{2}-m^{2}+i \epsilon\right) D^{\mathrm{c}}\right| y\right\rangle=-\langle x \mid y\rangle .
\end{aligned}
$$

Do resultado acima, podemos escrever

$$
\left(\Pi_{\mu}^{2}-m^{2}+i \epsilon\right) D^{\mathrm{c}}=-I
$$

e, assim, obter o valor de $D^{\mathrm{c}}$,

$$
D^{\mathrm{c}}=\left(-\Pi_{\mu}^{2}+m^{2}-i \epsilon\right)^{-1} \text {. }
$$

$D^{\mathrm{c}}$, por sua vez, é um operador de Bose e pode ser escrito como

$$
D^{\mathrm{c}}=i \int_{0}^{\infty} e^{-i \hat{\mathcal{H}}(\lambda, X, P)} d \lambda
$$

onde $\hat{\mathcal{H}}(\lambda, X, P)=\lambda\left(-\Pi_{\mu}^{2}+m^{2}-i \epsilon\right)$ e $\lambda$ é o tempo próprio.

Os passos feitos até agora podem ser resumidos em

$$
\begin{aligned}
D^{\mathrm{c}}\left(x_{\text {out }}, x_{\text {in }}\right) & =\left\langle x_{\text {out }}\left|D^{\mathrm{c}}\right| x_{\text {in }}\right\rangle \\
& =\left\langle x_{\text {out }}\left|\left(m^{2}-\Pi^{2}-i \epsilon\right)^{-1}\right| x_{\text {in }}\right\rangle \\
& =\left\langle x_{\text {out }}\left|i \int_{0}^{\infty} e^{-i \hat{\mathcal{H}}(\lambda, X, P)} d \lambda\right| x_{\text {in }}\right\rangle,
\end{aligned}
$$

onde $x_{\text {in }}$ e $x_{\text {out }}$ são, respectivamente, as posições iniciais e finais de $D^{\mathrm{c}}\left(x_{\text {out }}, x_{\text {in }}\right)$.

O próximo passo é escrever a última linha da equação acima como uma integral de 
trajetória. Para isso, vamos provar que

$$
D^{\mathrm{c}}\left(x_{\text {out }}, x_{\text {in }}\right)=\left\langle x_{\text {out }}\left|i \int_{0}^{\infty} e^{-i \hat{\mathcal{H}}(\lambda, X, P)} d \lambda\right| x_{\text {in }}\right\rangle
$$

pode ser escrita como

$$
D^{\mathrm{c}}\left(x_{\text {out }}, x_{\text {in }}\right)=i \int_{0}^{\infty} d \lambda_{0} \int D x D \lambda D p D \pi \exp \left[i \int_{0}^{1}[-\hat{\mathcal{H}}(\lambda, x, p)+p \dot{x}+\pi \dot{\lambda}] d \tau\right]
$$

Começamos escrevendo a eq. 2.4. como

$$
D^{\mathrm{c}}\left(x_{\text {out }}, x_{\text {in }}\right)=i \int_{0}^{\infty} d \lambda\left\langle x_{\text {out }}\left|\left[e^{-i \hat{\mathcal{H}}(\lambda, X, P) / N}\right]^{N}\right| x_{\text {in }}\right\rangle
$$

assim:

$$
D^{\mathcal{C}}\left(x_{\text {out }}, x_{\text {in }}\right)=i \int_{0}^{\infty} d \lambda\left\langle x_{\text {out }}\left|e^{-i \hat{\mathcal{H}}(\lambda, X, P) / N} e^{-i \hat{\mathcal{H}}(\lambda, X, P) / N} \cdots e^{-i \hat{\mathcal{H}}(\lambda, X, P) / N}\right| x_{\text {in }}\right\rangle .
$$

Agora é preciso transformar a integral sobre a variável $\lambda$ em uma integral sobre a trajetória $\lambda(\tau)$, parametrizada pelo parâmetro $\tau \in[0,1]$ e satisfazendo as condições de contorno $\lambda(0)=\lambda_{0}, x(0)=x_{\text {in }}$ e $x(1)=x_{\text {out }}$. Para isso, devemos inserir $N$ vezes a equação $I=\int_{-\infty}^{\infty} d \lambda_{m} \delta\left(\lambda_{m}-\lambda_{m-1}\right)$ e $N-1$ vezes a equação $I=\int_{-\infty}^{\infty} d x_{l}\left|x_{l}\right\rangle\left\langle x_{l}\right|$ na equação acima, com $m=1,2, \ldots, N$ e $l=1,2, \ldots, N-1$. Com isso, obtemos que

$$
\begin{aligned}
D^{\mathrm{c}}\left(x_{\text {out }}, x_{\text {in }}\right)= & i \int_{0}^{\infty} d \lambda_{0} \int_{-\infty}^{\infty} d x_{1} \ldots d x_{N-1} d \lambda_{1} \ldots d \lambda_{N} \\
& \times\left\langle x_{\text {out }}\left|e^{-i \hat{\mathcal{H}}\left(\lambda_{N}, X, P\right) / N}\right| x_{N-1}\right\rangle \delta\left(\lambda_{N}-\lambda_{N-1}\right) \\
& \times\left\langle x_{N-1}\left|e^{-i \hat{\mathcal{H}}\left(\lambda_{N-1}, X, P\right) / N}\right| x_{N-2}\right\rangle \delta\left(\lambda_{N-1}-\lambda_{N-2}\right) \\
& \times \ldots\left\langle x_{1}\left|e^{-i \hat{\mathcal{H}}\left(\lambda_{1}, X, P\right) / N}\right| x_{\text {in }}\right\rangle \delta\left(\lambda_{1}-\lambda_{0}\right),
\end{aligned}
$$

onde $x_{\text {out }}=x_{N}$ e $\lambda_{f}=\lambda_{N}$.

Para cada um dos termos $\left\langle x_{k}\left|e^{-i \hat{\mathcal{H}}\left(\lambda_{k}, X, P\right) / N}\right| x_{k-1}\right\rangle$ da equação acima, a exponencial pode ser expandida em série de Taylor:

$$
\left\langle x_{k}\left|e^{-i \hat{\mathcal{H}}\left(\lambda_{k}, X, P\right) / N}\right| x_{k-1}\right\rangle \approx\left\langle x_{k}\left|\hat{I}-i \hat{\mathcal{H}}\left(\lambda_{k}, X, P\right) / N\right| x_{k-1}\right\rangle .
$$

Através do ordenamento de Weyl, transformamos o operador $\hat{\mathcal{H}}\left(\lambda_{k}, X, P\right)$, no símbolo de Weyl $\mathcal{H}(\lambda, x, p)$. Para esse sistema o símbolo de Weyl é escrito como

$$
\mathcal{H}(\lambda, x, p)=\lambda\left(\mathcal{P}^{2}-m^{2}+i \epsilon\right)
$$

onde $\mathcal{P}_{\mu}=-p_{\mu}-A_{\mu}(x)$. 
O elemento de matriz da eq.(2.6) pode ser escrito em termos do ponto médio $\left(x_{k}+\right.$ $\left.x_{k-1}\right) / 2$ do símbolo de Wey - veja a ref. [59]. Com isso, obtém-se que

$$
\left\langle x_{k}\left|\hat{I}-i \hat{\mathcal{H}}\left(\lambda_{k}, X, P\right) / N\right| x_{k-1}\right\rangle=\frac{1}{(2 \pi)^{4}} \int\left[1-i \mathcal{H}\left(\lambda_{k}, \frac{x_{k}+x_{k-1}}{2}, p_{k}\right) / N\right] e^{i\left(x_{k}-x_{k-1}\right) p_{k}} d p_{k} .
$$

O termo entre chaves da equação acima pode ser escrito como uma exponencial aproximada:

$$
\left\langle x_{k}\left|\hat{I}-i \hat{\mathcal{H}}\left(\lambda_{k}, X, P\right) / N\right| x_{k-1}\right\rangle=\frac{1}{(2 \pi)^{4}} \int e^{-i \mathcal{H}\left(\lambda_{k}, \frac{x_{k}+x_{k-1}}{2}, p_{k}\right) / N} e^{i\left(x_{k}-x_{k-1}\right) p_{k}} d p_{k}+\mathcal{O}\left(\Delta T^{2}\right) .
$$

Desse modo, cada um dos termos da equação 2.5 com a forma $\left\langle x_{k}\left|\hat{I}-i \hat{\mathcal{H}}\left(\lambda_{k}, X, P\right) / N\right| x_{k-1}\right\rangle$ pode ser substituído pela equação acima. Obtém-se, assim, que

$$
\begin{aligned}
D^{\mathrm{C}}\left(x_{\text {out }}, x_{\text {in }}\right)= & i \int_{0}^{\infty} d \lambda_{0} \int_{-\infty}^{\infty} d x_{1} \ldots d x_{N-1} d \lambda_{1} \ldots d \lambda_{N} \frac{d p_{1}}{(2 \pi)^{4}} \ldots \frac{d p_{N}}{(2 \pi)^{4}} \\
& \times\left[e^{-i \mathcal{H}\left(\lambda_{N}, \frac{x_{N}+x_{N-1}}{2}, p_{N}\right) / N} e^{i\left(x_{N}-x_{N-1}\right) p_{N}} \delta\left(\lambda_{N}-\lambda_{N-1}\right)\right. \\
& \left.\times \ldots e^{-i \mathcal{H}\left(\lambda_{1}, \frac{x_{1}+x_{\text {in }}}{2}, p_{1}\right) / N} e^{i\left(x_{1}-x_{\text {in }}\right) p_{1}} \delta\left(\lambda_{1}-\lambda_{0}\right)\right] .
\end{aligned}
$$

A partir daqui será usada a notação $\int D x D \lambda D p D \pi$ em substituição a

$$
\int_{-\infty}^{\infty} d x_{1} \ldots d x_{N-1} d \lambda_{1} \ldots d \lambda_{N} \frac{d p_{1}}{(2 \pi)^{4}} \ldots \frac{d p_{N}}{(2 \pi)^{4}} \frac{d \pi_{1}}{2 \pi} \ldots \frac{d \pi_{N}}{2 \pi} .
$$

Inserindo a relação

$$
\delta\left(\lambda_{k}-\lambda_{k-1}\right)=\frac{1}{2 \pi} \int_{-\infty}^{\infty} e^{i \pi_{k}\left(\lambda_{k}-\lambda_{k-1}\right)} d \pi_{k}
$$

na equação acima, obtém-se que

$$
\begin{aligned}
D^{\mathrm{c}}\left(x_{\text {out }}, x_{\text {in }}\right)= & i \int_{0}^{\infty} d \lambda_{0} \int D x D \lambda D p D \pi \\
& \times \exp \left(-i \mathcal{H}\left(\lambda_{N}, \frac{x_{N}+x_{N-1}}{2}, p_{N}\right) / N\right) \exp \left[i\left(x_{N}-x_{N-1}\right) p_{N}\right] \exp \left[i \pi_{N}\left(\lambda_{N}-\lambda_{N-1}\right)\right] \\
& \times \ldots \exp \left(-i \mathcal{H}\left(\lambda_{1}, \frac{x_{1}+x_{\text {in }}}{2}, p_{1}\right) / N\right) \exp \left[i\left(x_{1}-x_{\text {in }}\right) p_{1}\right] \exp \left[i \pi_{1}\left(\lambda_{1}-\lambda_{0}\right)\right],
\end{aligned}
$$

que pode ser escrita como 


$$
\begin{aligned}
D^{\mathrm{c}}\left(x_{\text {out }}, x_{\text {in }}\right)= & i \int_{0}^{\infty} d \lambda_{0} \int D x D \lambda D p D \pi \exp \left[-i \mathcal{H}\left(\lambda_{N}, \frac{x_{N}+x_{N-1}}{2}, p_{N}\right) / N+i\left(x_{N}-x_{N-1}\right) p_{N}\right. \\
& \left.+i \pi_{N}\left(\lambda_{N}-\lambda_{N-1}\right)+\ldots-i \mathcal{H}\left(\lambda_{1}, \frac{x_{1}+x_{\text {in }}}{2}, p_{1}\right) / N+i\left(x_{1}-x_{\text {in }}\right) p_{1}+i \pi_{1}\left(\lambda_{1}-\lambda_{0}\right)\right],
\end{aligned}
$$

e, então, reescrita novamente como

$$
\begin{aligned}
D^{\mathrm{c}}\left(x_{\text {out }}, x_{\text {in }}\right)= & i \int_{0}^{\infty} d \lambda_{0} \int D x D \lambda D p D \pi \\
& \times \exp \left[i \sum_{k=1}^{N}\left[p_{k} \frac{\left(x_{k}-x_{k-1}\right)}{\Delta \tau}-\mathcal{H}\left(\lambda_{k}, \frac{x_{k}+x_{k-1}}{2}, p_{k}\right)+\pi_{k} \frac{\left(\lambda_{k}-\lambda_{k-1}\right)}{\Delta \tau}\right] \Delta \tau\right] .
\end{aligned}
$$

No limite em que $N \rightarrow \infty$ (e, portanto, $\Delta \tau \rightarrow 0)$, a equação acima se torna, finalmente:

$$
D^{\mathrm{c}}\left(x_{\text {out }}, x_{\text {in }}\right)=i \int_{0}^{\infty} d \lambda_{0} \int D x D \lambda D p D \pi \exp \left[i \int_{0}^{1}[p \dot{x}-\mathcal{H}(\lambda, x, p)+\pi \dot{\lambda}] d \tau\right] .
$$

Finalizamos com isso a prova de que a eq. 2.4 pode ser escrita como uma integral de trajetória.

Aplicando este resultado ao nosso sistema e usando a eq.(2.7), encontramos:

$$
\begin{aligned}
D^{C}\left(x_{\text {out }}, x_{\text {in }}\right)= & i \int_{0}^{\infty} d \lambda_{0} \int D x D \lambda D p D \pi \\
& \times \exp \left[i \int_{0}^{1}\left[p \dot{x}+\lambda\left(\mathcal{P}^{2}-m^{2}\right)+\pi \dot{\lambda}\right] d \tau\right] .
\end{aligned}
$$

Para obter a integral de trajetória em sua forma Lagrangiana, é necessário, na equação acima, integrar sobre o momento $p$. Para isto, é feita uma translação no momento, $p_{\mu} \rightarrow p_{\mu}+\tilde{p}_{\mu}$, onde $\tilde{p}_{\mu}$ é o momento clássico.

O momento clássico, por sua vez, é obtido a partir da equação

$$
\frac{\partial \mathcal{H}}{\partial p_{\alpha}}=\dot{x}^{\alpha}=-2 \lambda\left[p^{\alpha}+g A^{\alpha}(x)\right] \text {. }
$$

Com isso, obtemos

$$
\tilde{p}_{\mu}=-g A_{\mu}(x)-\frac{\dot{x}_{\mu}}{2 \lambda}
$$

Com estes resultados vamos realizar as seguintes substituições na eq.(2.8):

$$
p_{\mu} \rightarrow p_{\mu}-g A_{\mu}(x)-\frac{\dot{x}_{\mu}}{2 \lambda} \quad, \quad \lambda=e / 2
$$

A substituição correspondente à translação no momento nos fornece uma integral em 
que é possível separar o termo do momento do restante dos termos:

$$
\begin{aligned}
D^{\mathrm{C}}\left(x_{\text {out }}, x_{\mathrm{in}}\right)= & \frac{i}{2} \int_{0}^{\infty} d e_{0} \int D x D \lambda D p D \pi \\
& \times \exp \left[i \int_{0}^{1}\left[\frac{e p^{2}}{2}-\frac{\dot{x}^{2}}{2 e}-\frac{e m^{2}}{2}-g \dot{x} A(x)+\frac{\pi \dot{e}}{2}\right] d \tau\right] .
\end{aligned}
$$

Com isso, a integral acima pode ser separada em três partes. A primeira parte é a medida de integração $\mathcal{M}(e)$,

$$
\mathcal{M}(e)=\int D p \exp \left[\frac{i}{2} \int_{0}^{1} e p^{2} d \tau\right],
$$

a segunda parte é a ação invariante por calibre da partícula,

$$
S=-\int_{0}^{1}\left[\frac{\dot{x}^{2}}{2 e}+\frac{e m^{2}}{2}+g \dot{x} A(x)\right] d \tau,
$$

com as transformações de calibre na forma:

$$
x(\tau) \rightarrow x(f(\tau)), \quad e(\tau) \rightarrow e(f(\tau)) \dot{f}(\tau), \quad f(0)=0, \quad f(1)=1
$$

as quais, em termos infinitesimais, podem ser escritas como:

$$
\delta x=\dot{x} \epsilon, \quad \delta e=\frac{d}{d \tau}(e \epsilon), \quad \epsilon(0)=\epsilon(1)=0 .
$$

A terceira parte é um termo de fixação de calibre,

$$
S_{G F}=-\int_{0}^{1} \pi \dot{e} d \tau,
$$

que fixa $\dot{e}=0$.

A obtenção da eq. 2.9 e a sua separação em três termos encerra este capítulo, no qual apresentamos o método para obter a ação clássica de um sistema relativamente simples. Nos próximos capítulos realizaremos essencialmente os mesmos processos aqui realizados. Entretanto, por se tratarem de sistemas fermiônicos serão necessárias algumas adaptações, conforme serão discutidas a seguir. 


\section{CAPÍTULO 3}

\section{Modelo pseudoclássico de uma partícula de Dirac que in- terage com o campo eletromagnético}

Neste capítulo também reproduzimos as contas da referência [1]. Este passo é importante para que possamos saber aplicar o método de Fradkin e Gitman em sistemas de férmions. Alguns dos processos do capítulo anterior devem ser modificados e outros são acrescentados. Como já feito no artigo [1], vamos obter a ação pseudoclássica do sistema descrito pela seguinte equação de Dirac modificada:

$$
\left[\left(i \partial_{\mu}-g A_{\mu}(x)\right) \gamma^{\mu}-m\right] \Psi(x)=0
$$

Ela descreve o sistema composto por uma partícula relativística com spin $1 / 2$ que interage com um campo eletromagnético externo. A métrica que usamos é a $\eta_{\mu v}=$ $\operatorname{diag}(1,-1,-1,-1), A_{\mu}(x)$ é o potencial eletromagnético externo, com $\mu=0,1,2,3, g$ é a carga elétrica e $\gamma^{\mu}$ são as matrizes de Dirac que satisfazem a relação $\left[\gamma^{\mu}, \gamma^{\nu}\right]_{+}=2 \eta^{\mu \nu}$. Além disso, usamos também a matriz $\gamma^{5}$ definida como $\gamma^{5}=\gamma^{0} \gamma^{1} \gamma^{2} \gamma^{3}$. Neste capítulo são usados os mesmos operadores e autovetores definidos nas equações $(2.2)$ do capítulo 2 .

Diferentemente do sistema do capítulo anterior, a partícula tratada neste capítulo possui spin e é um férmion. Por essas razões, utilizaremos a álgebra de Grassmann e dessa forma, o modelo que iremos obter ao final do método é chamado de modelo pseudoclássico. Os geradores dessa álgebra serão chamados de $a_{1}, \ldots, a_{n}$. Eles anticomutam e o quadrado de um gerador é igual a zero:

$$
a_{k} a_{l}+a_{l} a_{k}=0, \quad a_{k} a_{k}=0, \quad k, l=1, \ldots, n .
$$

Estes geradores formam polinômios, os quais, quando são compostos por um número ímpar (par) de geradores da álgebra de Grassmann, são denominados variáveis de Grassmann ímpares (pares). Variáveis ímpares anticomutam com variáveis ímpares; por outro lado, variáveis pares comutam com variáveis pares e ímpares.

A derivada parcial de uma variável ímpar pode ser feita de duas formas. Supondo 
que $\chi$ seja uma variável de Grassmann ímpar, uma das formas de se realizar uma derivada em $\chi$ é a chamada derivada pela esquerda, $\frac{\partial_{l}}{\partial \chi}$, caracterizada pelo subíndice l. Neste caso, a variável que será derivada deve ser deslocada para a posição mais à esquerda do termo do qual ela faz parte e só então derivada. Esse deslocamento pode ou não gerar uma mudança no sinal do termo que está sendo derivado, devido a anticomutatividade das variáveis ímpares. A segunda forma de se realizar a derivada é a derivada pela direita, $\frac{\partial_{r}}{\partial \chi}$, caracterizada pelo subíndice $r$. Nesta derivada, a variável que será derivada deve ser deslocada para a posição mais à direita do termo do qual ela pertence antes de ser derivada.

Estas são as propriedades da álgebra de Grassmann que serão utilizadas neste capítulo. Outras propriedades podem ser encontradas no capítulo 6 da referência bibliográfica [60]. Variáveis pares e ímpares são elementos da álgebra de Berezin, F. A. Berezin estendeu a álgebra de Grassmann nos fornecendo importantes resultados, alguns deles serão usados na segunda parte do capítulo 4. A referência [57], e em especial o seu apêndice $\mathrm{D}$, trata da álgebra de Berezin.

\subsection{Ação pseudoclássica}

Para obtermos a ação pseudoclássica do sistema, iniciamos escrevendo a eq.3.1) em termos da função de Green causal do sistema, $S^{C}(x, y)$,

$$
\left[\left(i \partial_{\mu}-g A_{\mu}(x)\right) \gamma^{\mu}-m\right] S^{C}(x, y)=-\delta^{4}(x-y)
$$

É conveniente, no entanto, usarmos $\tilde{S}^{C}(x, y)=S^{C}(x, y) \gamma^{5}$ no lugar de $S^{C}(x, y)$. Para isso, multiplicamos a equação acima por $\gamma^{5}$, da seguinte maneira:

$$
\gamma^{5}\left[\left(i \partial_{\mu}-g A_{\mu}(x)\right) \gamma^{\mu}-m\right] S^{C}(x, y) \gamma^{5}=-\gamma^{5} \delta^{4}(x-y) \gamma^{5}
$$

Além disso, no lugar das matrizes $\gamma^{\mu}$ e $\gamma^{5}$ usaremos as matrizes $\Gamma^{n}, \operatorname{com} n=0,1,2,3,4$ :

$$
\Gamma^{\mu}=\gamma^{5} \gamma^{\mu}, \Gamma^{4}=\gamma^{5}, \mu=0,1,2,3
$$

Estas matrizes satisfazem a relação:

$$
\left[\Gamma^{n}, \Gamma^{m}\right]_{+}=2 \eta^{n m}, \eta^{n m}=\operatorname{diag}(1,-1,-1,-1,-1) .
$$

Com essas transformações, a eq.(3.2) é escrita como

$$
\left[\left(i \partial_{\mu}-g A_{\mu}(x)\right) \Gamma^{\mu}-\Gamma^{4} m+i \epsilon\right] \tilde{S}^{C}(x, y)=\delta^{4}(x-y) .
$$


Segundo [58], é possível escrever a função $\tilde{S}_{\alpha \beta}^{C}(x, y)$ como um elemento de matriz do operador $\tilde{S}_{\alpha \beta}^{C}$,

$$
\tilde{S}_{\alpha \beta}^{C}(x, y)=\left\langle x\left|\tilde{S}_{\alpha \beta}^{C}\right| y\right\rangle,
$$

onde os subíndices $\alpha, \beta$ são índices espinoriais e serão desconsiderados a partir daqui. De forma semelhante ao feito no capítulo anterior, obtemos o valor de $\tilde{S}^{C}$ :

$$
\tilde{S}^{C}=\left(\Pi_{\mu} \Gamma^{\mu}-\Gamma^{4} m\right)^{-1},
$$

onde $\left(i \partial_{\mu}-g A_{\mu}(x)\right)$ é escrito como o operador $\Pi_{\mu}=\left(-P_{\mu}-g A_{\mu}(X)\right)$.

$\tilde{S}^{C}$ é um operador de Fermi por conter variáveis de Grassmann. Consideramos que as matrizes $\Gamma^{n}$ também são operadores de Fermi. Gitman e Fradkin desenvolveram uma fórmula para escrever um operador de Fermi como a integral de uma exponencial:

$$
A^{-1}=\int_{0}^{\infty} d \lambda \int e^{i\left[\lambda\left(A^{2}+i \epsilon\right)+\chi A\right]} d \chi
$$

onde $A$ é um operador de Fermi ímpar, $\lambda$ é uma variável par e $\chi$ uma variável ímpar que, por definição, anticomuta $\operatorname{com} A$ - veja a Ref. [1]. Mesmo que uma variável de Grassmann elevada ao quadrado seja igual a zero, podemos elevar $A$ ao quadrado já que ela é uma combinação de operadores escritos com as variáveis de Grassmann e essa combinação elevada ao quadrado não é necessariamente zero. Os passos feitos até agora podem se resumir em:

$$
\begin{aligned}
\tilde{S}^{C}\left(x_{\text {out }}, x_{\text {in }}\right) & =\left\langle x_{\text {out }}\left|\tilde{S}^{C}\right| x_{\text {in }}\right\rangle \\
& =\left\langle x_{\text {out }}\left|\left(N-\Gamma^{4} m\right)^{-1}\right| x_{\text {in }}\right\rangle \\
& =\left\langle x_{\text {out }}\left|\int_{0}^{\infty} d \lambda \int e^{-i \hat{\mathcal{H}}(\lambda, \chi, X, P)} d \chi\right| x_{\text {in }}\right\rangle,
\end{aligned}
$$

onde

$$
\begin{aligned}
-i \hat{\mathcal{H}}(\lambda, \chi, X, P) & =i\left[\lambda\left(A^{2}+i \epsilon\right)+\chi A\right] \\
& =i\left[\lambda\left(\left(\Pi_{\mu} \Gamma^{\mu}-\Gamma^{4} m\right)^{2}+i \epsilon\right)+\chi\left(\Pi_{\mu} \Gamma^{\mu}-\Gamma^{4} m\right)\right] \\
& =i\left[\lambda\left(\left(\Pi_{\mu} \Gamma^{\mu}\right)^{2}-m^{2}\right)+\chi\left(\Pi_{\mu} \Gamma^{\mu}-\Gamma^{4} m\right)\right] \\
& =i\left[\lambda\left(\Pi^{2}-\frac{i g}{2} \mathbb{F}_{\mu \nu} \Gamma^{\mu} \Gamma^{v}-m^{2}\right)+\chi\left(\Pi_{\mu} \Gamma^{\mu}-\Gamma^{4} m\right)\right] .
\end{aligned}
$$

Para obter o resultado acima usamos $\left[\Gamma^{m}, \Gamma^{n}\right]_{+}=2 \eta^{n m}, \epsilon=0$ e $\mathbb{F}_{\mu \nu}=\partial_{\mu} A_{\nu}-\partial_{\nu} A_{\mu}$.

Tal como no capítulo 2, vamos provar que a última linha da equação (3.3) pode 
ser escrita como uma integral de trajetória. Muitos dos passos que devemos fazer são semelhantes aos passos da prova do capítulo anterior. No entanto, neste caso temos uma integração sobre a variável $\chi$ e, devemos reescrever a Hamiltoniana de modo que as matrizes $\Gamma^{n}$ não nos atrapalhe.

Primeiro provaremos que

$$
\tilde{S}^{C}\left(x_{\text {out }}, x_{\text {in }}\right)=\int_{0}^{\infty} d \lambda \int\left\langle x_{\text {out }}\left|e^{-i \hat{\mathcal{H}}(\lambda, \chi, X, P)}\right| x_{\text {in }}\right\rangle d \chi
$$

pode ser escrita como

$$
\begin{aligned}
\tilde{S}^{C}\left(x_{\text {out }}, x_{\text {in }}\right)= & \int_{0}^{\infty} d \lambda_{0} \int_{0}^{\infty} d \chi_{0} \int D x D \lambda D \chi D p D \pi D v \\
& \times \exp \left[i \int_{0}^{1}[-\hat{\mathcal{H}}(\lambda, \chi, x, p)+p \dot{x}+\pi \dot{\lambda}+v \dot{\chi}] d \tau\right] .
\end{aligned}
$$

O primeiro passo será escrever a eq. 3.4 como

$$
\tilde{S}^{C}\left(x_{\text {out }}, x_{\text {in }}\right)=\int_{0}^{\infty} d \lambda \int\left\langle x_{\text {out }}\left|\left[e^{-i \hat{\mathcal{H}}(\lambda, \chi, X, P) / N}\right]^{N}\right| x_{\text {in }}\right\rangle d \chi
$$

Assim,

$$
\tilde{S}^{C}\left(x_{\text {out }}, x_{\text {in }}\right)=\int_{0}^{\infty} d \lambda \int d \chi\left\langle x_{\text {out }}\left|e^{-i \hat{\mathcal{H}}(\lambda, \chi, X, P) / N} e^{-i \hat{\mathcal{H}}(\lambda, \chi, X, P) / N} \ldots e^{-i \hat{\mathcal{H}}(\lambda, \chi, X, P) / N}\right| x_{\text {in }}\right\rangle .
$$

Para que a integral sobre as variáveis $\lambda$ e $\chi$ se tornem integrais sobre as trajetórias $\lambda(\tau) \mathrm{e}$ $\chi(\tau)$, parametrizadas pelo parâmetro $\tau \in[0,1]$ e satisfazendo as condições de contorno $\lambda(0)=\lambda_{0}, \chi(0)=\chi_{0}, x(0)=x_{\text {in }}$ e $x(1)=x_{\text {out }}$., inserimos na equação acima as equações $I=\int_{-\infty}^{\infty} d \lambda_{m} \delta\left(\lambda_{m}-\lambda_{m-1}\right)$ e $I=\int_{-\infty}^{\infty} d \chi_{l} \delta\left(\chi_{l}-\chi_{l-1}\right), l, m=1,2, \ldots, N$, e $I=\int_{-\infty}^{\infty} d x_{l}\left|x_{l}\right\rangle\left\langle x_{l}\right|$, $l=1,2, \ldots, N-1$. Obtemos, com isso,

$$
\begin{aligned}
& \tilde{S}^{C}\left(x_{\text {out }}, x_{\text {in }}\right)=\int_{0}^{\infty} d \lambda_{0} \int_{0}^{\infty} d \chi_{0} \int_{-\infty}^{\infty} d x_{1} \ldots d x_{N-1} d \lambda_{1} \ldots d \lambda_{N} d \chi_{1} \ldots d \chi_{N} \\
& \times\left\langle x_{\text {out }}\left|e^{-i \hat{\mathcal{H}}\left(\lambda_{N}, \chi_{N}, X, P\right) / N}\right| x_{N-1}\right\rangle \delta\left(\lambda_{N}-\lambda_{N-1}\right) \delta\left(\chi_{N}-\chi_{N-1}\right) \\
& \times \ldots\left\langle x_{1}\left|e^{-i \hat{\mathcal{H}}\left(\lambda_{1}, \chi_{1}, X, P\right) / N}\right| x_{\mathrm{in}}\right\rangle \delta\left(\lambda_{1}-\lambda_{0}\right) \delta\left(\chi_{1}-\chi_{0}\right),
\end{aligned}
$$

onde $x_{\text {out }}=x_{N}, \lambda_{f}=\lambda_{N}$ e $\chi_{f}=\chi_{N}$.

Para cada um dos termos $\left\langle x_{k}\left|e^{-i \hat{\mathcal{H}}\left(\lambda_{k}, \chi_{k}, X, P\right) / N}\right| x_{k-1}\right\rangle$ da equação acima, expandimos a exponencial em série de Taylor,

$$
\left\langle x_{k}\left|e^{-i \hat{\mathcal{H}}\left(\lambda_{k}, \chi_{k}, X, P\right) / N}\right| x_{k-1}\right\rangle \approx\left\langle x_{k}\left|\hat{I}-i \hat{\mathcal{H}}\left(\lambda_{k}, \chi_{k}, X, P\right) / N\right| x_{k-1}\right\rangle .
$$


O operador $\hat{\mathcal{H}}(\lambda, \chi, X, P)$ possui o seguinte símbolo de Weyl:

$$
\mathcal{H}(\lambda, \chi, x, p)=-\left[\lambda\left(\mathcal{P}^{2}-\frac{i g}{2} \mathbb{F}_{\mu \nu} \Gamma^{\mu} \Gamma^{\nu}-m^{2}\right)+\chi\left(\mathcal{P}_{\mu} \Gamma^{\mu}-\Gamma^{4} m\right)\right]
$$

onde $\mathcal{P}_{\mu}=-p_{\mu}-A_{\mu}(x)$.

O elemento de matriz da eq.(3.6) é escrito em termos do ponto médio $\left(x_{k}+x_{k-1}\right) / 2$ do símbolo de Weyl- veja a ref. [59]:

$$
\left\langle x_{k}\left|\hat{I}-i \hat{\mathcal{H}}\left(\lambda_{k}, \chi_{k}, X, P\right) / N\right| x_{k-1}\right\rangle=\frac{1}{(2 \pi)^{4}} \int\left[1-i \mathcal{H}\left(\lambda_{k}, \chi_{k}, \frac{x_{k}+x_{k-1}}{2}, p_{k}\right) / N\right] e^{i\left(x_{k}-x_{k-1}\right) p_{k}} d p_{k}
$$

O termo entre chaves da equação acima pode ser escrito como uma exponencial:

$$
\left\langle x_{k}\left|\hat{I}-i \mathcal{H}\left(\lambda_{k}, X, P\right) / N\right| x_{k-1}\right\rangle=\frac{1}{(2 \pi)^{4}} \int e^{-i \mathcal{H}\left(\lambda_{k}, \chi_{k}, \frac{x_{k}+x_{k-1}}{2}, p_{k}\right) / N} e^{i\left(x_{k}-x_{k-1}\right) p_{k}} d p_{k}+\mathcal{O}\left(\Delta T^{2}\right),
$$

e a sua substituição na eq. (3.5) nos fornece

$$
\begin{aligned}
\tilde{S}^{C}\left(x_{\text {out }}, x_{\text {in }}\right)= & \int_{0}^{\infty} d \lambda_{0} \int_{0}^{\infty} d \chi_{0} \int_{-\infty}^{\infty} d x_{1} \ldots d x_{N-1} d \lambda_{1} \ldots d \lambda_{N} d \chi_{1} \ldots d \chi_{N} \frac{d p_{1}}{(2 \pi)} \ldots \frac{d p_{N}}{(2 \pi)^{4}} \\
& \times\left[e^{-i \mathcal{H}\left(\lambda_{N}, \chi_{N}, \frac{x_{N}+x_{N-1}}{2}, p_{N}\right) / N} e^{i\left(x_{N}-x_{N-1}\right) p_{N}} \delta\left(\lambda_{N}-\lambda_{N-1}\right) \delta\left(\chi_{N}-\chi_{N-1}\right) \ldots\right. \\
& \left.\times \ldots e^{-i \mathcal{H}\left(\lambda_{1}, \chi_{1}, \frac{x_{1}+x_{\text {in }}}{2}, p_{1}\right) / N} e^{i\left(x_{1}-x_{\text {in }}\right) p_{1}} \delta\left(\lambda_{1}-\lambda_{0}\right) \delta\left(\chi_{1}-\chi_{0}\right)\right] .
\end{aligned}
$$

Os termos

$$
\begin{aligned}
& \delta\left(\lambda_{k}-\lambda_{k-1}\right)=\frac{1}{2 \pi} \int_{-\infty}^{\infty} e^{i \pi_{k}\left(\lambda_{k}-\lambda_{k-1}\right)} d \pi_{k}, \\
& \delta\left(\chi_{k}-\chi_{k-1}\right)=\frac{1}{2 \pi} \int_{-\infty}^{\infty} e^{i v_{k}\left(\chi_{k}-\chi_{k-1}\right)} d v_{k},
\end{aligned}
$$

nos permite escrever $\tilde{S}^{C}\left(x_{\text {out }}, x_{\text {in }}\right)$ como o produto de exponenciais:

$$
\begin{aligned}
& \tilde{S}^{C}\left(x_{\text {out }}, x_{\text {in }}\right)=\int_{0}^{\infty} d \lambda_{0} \int_{0}^{\infty} d \chi_{0} \int D x D \lambda D \chi D p D \pi D v \\
& \times\left[e^{-i \mathcal{H}\left(\lambda_{N}, \frac{x_{N}+x_{N-1}}{2}, p_{N}\right) / N} e^{i\left(x_{N}-x_{N-1}\right) p_{N}} e^{i \pi_{N}\left(\lambda_{N}-\lambda_{N-1}\right)} e^{i v_{N}\left(\chi_{N}-\chi_{N-1}\right)} \ldots\right. \\
& \left.\times e^{-i \mathcal{H}\left(\lambda_{1}, \frac{x_{1}+x_{\text {in }}}{2}, p_{1}\right) / N} e^{i\left(x_{1}-x_{\text {in }}\right) p_{1}} e^{i \pi_{1}\left(\lambda_{1}-\lambda_{0}\right)} e^{i v_{1}\left(\chi_{1}-\chi_{0}\right)}\right] .
\end{aligned}
$$


Dessa forma, podemos reordenar $\tilde{S}^{C}\left(x_{\text {out }}, x_{\text {in }}\right)$ como uma única exponencial:

$$
\begin{aligned}
& \tilde{S}^{C}\left(x_{\mathrm{out}}, x_{\mathrm{in}}\right)=\int_{0}^{\infty} d \lambda_{0} \int_{0}^{\infty} d \chi_{0} \int D x D \lambda D \chi D p D \pi D v \\
& \times \exp \left[-i \mathcal{H}\left(\lambda_{N}, \chi_{N}, \frac{x_{N}+x_{N-1}}{2}, p_{N}\right) / N+i\left(x_{N}-x_{N-1}\right) p_{N}\right. \\
& +i \pi_{N}\left(\lambda_{N}-\lambda_{N-1}\right)+i v_{N}\left(\chi_{N}-\chi_{N-1}\right) \ldots-i \mathcal{H}\left(\lambda_{1}, \chi_{1}, \frac{x_{1}+x_{\mathrm{in}}}{2}, p_{1}\right) / N \\
& \left.+i\left(x_{1}-x_{\mathrm{in}}\right) p_{1}+i \pi_{1}\left(\lambda_{1}-\lambda_{0}\right)+i v_{1}\left(\chi_{1}-\chi_{0}\right)\right]
\end{aligned}
$$

e em seguida, juntar todos os termos da exponencial em um somatório:

$$
\begin{aligned}
& \tilde{S}^{C}\left(x_{\text {out }}, x_{\text {in }}\right)=\int_{0}^{\infty} d \lambda_{0} \int_{0}^{\infty} d \chi_{0} \int D x D \lambda D \chi D p D \pi D v \\
& \times \exp \left[i \sum_{k=1}^{N}\left[p_{k} \frac{\left(x_{k}-x_{k-1}\right)}{\Delta \tau}-\mathcal{H}\left(\lambda_{k}, \chi_{k}, \frac{x_{k}+x_{k-1}}{2}, p_{k}\right)+\pi_{k} \frac{\left(\lambda_{k}-\lambda_{k-1}\right)}{\Delta \tau}+v_{k} \frac{\left(\chi_{k}-\chi_{k-1}\right)}{\Delta \tau}\right] \Delta \tau\right] .
\end{aligned}
$$

Tomando o limite em que $N \rightarrow \infty$ (e, portanto, $\Delta \tau \rightarrow 0$ ), obtemos:

$$
\begin{aligned}
& \tilde{S}^{C}\left(x_{\mathrm{out}}, x_{\mathrm{in}}\right)=\int_{0}^{\infty} d \lambda_{0} \int_{0}^{\infty} d \chi_{0} \int D x D \lambda D \chi D p D \pi D v \\
& \times \exp \left[i \int_{0}^{1}[p \dot{x}-\mathcal{H}(\lambda, \chi, x, p)+\pi \dot{\lambda}+v \dot{\chi}] d \tau\right] .
\end{aligned}
$$

Concluímos com isso a prova de que a eq. (3.4 pode ser escrita como uma integral de trajetória. Este resultado também será utilizado no capítulo 4.

Aplicando este resultado ao nosso sistema, e usando a eq. 3.7, obtemos que

$$
\begin{aligned}
& \tilde{S}^{C}\left(x_{\text {out }}, x_{\text {in }}\right)=\int_{0}^{\infty} d \lambda_{0} \int_{0}^{\infty} d x_{0} \int D x D \lambda D \chi D p D \pi D \nu \\
& \times \exp \left\{i \int_{0}^{1}\left[-\lambda\left[m^{2}-\mathcal{P}^{2}+\frac{i g}{2} \mathbb{F}_{\mu \nu} \Gamma^{\mu} \Gamma^{\nu}\right]-\left(\mathcal{P}_{\mu} \Gamma^{\mu}-\Gamma^{4} m\right) \chi+p \dot{x}+\pi \dot{\lambda}+v \dot{\chi}\right] d \tau\right\} .
\end{aligned}
$$

A equação acima depende das matrizes $\Gamma^{\alpha}$, para que isso não ocorra, usamos que $\Gamma^{\alpha}$ pode ser escrita como:

$$
\frac{\delta_{l}}{\delta \rho_{\alpha}}\left[\left.T\left(\exp \int_{0}^{1} \rho_{n}(\tau) \Gamma^{n} d \tau\right)\right|_{\rho=0}\right]=\Gamma^{\alpha}\left[\left.T\left(\exp \int_{0}^{1} \rho_{n}(\tau) \Gamma^{n} d \tau\right)\right|_{\rho=0}\right]=\Gamma^{\alpha}
$$

onde $T\left(\exp \int_{0}^{1} \rho_{n}(\tau) \Gamma^{n} d \tau\right)$ é uma exponencial ordenada no tempo, esse ordenamento é necessário porque $\rho_{n}(\tau) \Gamma^{n}$ não comuta com $\rho_{n}\left(\tau^{\prime}\right) \Gamma^{n}$ quando $\tau \neq \tau^{\prime}$. Essa forma de escrever $\Gamma^{\alpha}$ foi pensada por Fradkin e Gitman. Escrever $\Gamma^{\alpha}$ dessa forma na equação 
(3.8) resulta em:

$$
\begin{aligned}
\tilde{S}^{C}\left(x_{\text {out }}, x_{\text {in }}\right)= & \int_{0}^{\infty} d \lambda_{0} \int_{0}^{\infty} d \chi_{0} \int D x D \lambda D \chi D p D \pi D v \\
& \times \exp \left\{i \int _ { 0 } ^ { 1 } \left[-\lambda\left[m^{2}-\mathcal{P}^{2}+\frac{i g}{2} \mathbb{F}_{\mu \nu} \frac{\delta_{l}}{\delta \rho_{\mu}} \frac{\delta_{l}}{\delta \rho_{\nu}}\right]\right.\right. \\
& \left.\left.-\left(\mathcal{P}_{\mu} \frac{\delta_{l}}{\delta \rho_{\mu}}-\frac{\delta_{l}}{\delta \rho_{4}} m\right) \chi+p \dot{x}+\pi \dot{\lambda}+v \dot{\chi}\right] d \tau\right\} T \exp \left[\left.\int_{0}^{1} \rho_{n}(\tau) \Gamma^{n} d \tau\right|_{\rho=0}\right],
\end{aligned}
$$

onde $\rho_{n}(\tau)$, com $n=0, \ldots, 4$, são cinco fontes ímpares que anticomutam com as matrizes $\Gamma^{\alpha}$ por definição.

No apêndice provamos que o último termo da equação (3.9):

$$
T \exp \left[\left.\int_{0}^{1} \rho_{n}(\tau) \Gamma^{n} d \tau\right|_{\rho=0}\right],
$$

pode ser escrito como

$$
\begin{aligned}
T \exp \left[\left.\int_{0}^{1} \rho_{n}(\tau) \Gamma^{n} d \tau\right|_{\rho=0}\right] & =\exp \left[i \Gamma^{n} \frac{\partial_{l}}{\partial \theta^{n}}\right] \\
& \times\left.\int \exp \left[\int_{0}^{1}\left(\psi_{n} \dot{\psi}^{n}-2 i \rho_{n} \psi^{n}\right) d \tau+\psi_{n}(1) \psi^{n}(0)\right] D \psi\right|_{\theta=0}
\end{aligned}
$$

Aplicando este resultado na eq.3.9 e escrevendo a exponencial como produtos de exponenciais, obtemos que

$$
\begin{aligned}
& \tilde{S}^{C}\left(x_{\text {out }}, x_{\text {in }}\right)=\exp \left[i \Gamma^{n} \frac{\partial_{l}}{\partial \theta^{n}}\right] \int_{0}^{\infty} d \lambda_{0} \int_{0}^{\infty} d \chi_{0} \int D x D \lambda D \chi D p D \pi D \nu D \psi \\
& \times \exp \left\{i \int_{0}^{1}\left[-\lambda\left(m^{2}-\mathcal{P}^{2}\right) d \tau\right]\right\} \exp \left[i \int_{0}^{1}\left(\frac{i g}{2} F_{\mu \nu} \frac{\delta_{l}}{\delta \rho_{\mu}} \frac{\delta_{l}}{\delta \rho_{\nu}}\right) d \tau\right] \\
& \times \exp \left[i \int_{0}^{1}\left(-\mathcal{P}_{\mu} \frac{\delta_{l}}{\delta \rho_{\mu}} \chi\right) d \tau\right] \exp \left[i \int_{0}^{1}\left(\frac{\delta_{l}}{\delta \rho_{5}} m \chi\right) d \tau\right] \\
& \times \exp \left[i \int_{0}^{1}(p \dot{x}+\pi \dot{\lambda}+v \dot{\chi}) d \tau\right] \exp \left(-i \int \rho^{n}\left(\tau_{1}\right) 2 \psi_{n}\left(\tau_{1}\right) d \tau_{1}\right) \\
& \times\left.\int \exp \left[\int_{0}^{1} \psi_{n} \dot{\psi}^{n} d \tau+\psi_{n}(1) \psi^{n}(0)\right]\right|_{\theta=0} .
\end{aligned}
$$


As exponenciais que possuem derivadas funcionais são expandidos em série de Taylor:

$$
\begin{aligned}
& \tilde{S}^{C}\left(x_{\text {out }}, x_{\text {in }}\right)=\exp \left[i \Gamma^{n} \frac{\partial_{l}}{\partial \theta^{n}}\right] \int_{0}^{\infty} d \lambda_{0} \int_{0}^{\infty} d \chi_{0} \int D x D \lambda D \chi D p D \pi D \nu D \psi \\
& \times \exp \left\{i \int_{0}^{1}\left[-\lambda\left(m^{2}-\mathcal{P}^{2}\right) d \tau\right]\right\} \sum_{m=0}^{\infty}\left[\frac{1}{m !}\left(-i \int_{0}^{1} \frac{i g}{2} F_{\mu \nu} \frac{\delta_{l}}{\delta \rho_{\mu}} \frac{\delta_{l}}{\delta \rho_{\nu}} d \tau\right)^{m}\right] \\
& \times \sum_{n=0}^{\infty}\left[\frac{1}{n !}\left(-i \int_{0}^{1} \mathcal{P}_{\mu} \frac{\delta_{l}}{\delta \rho_{\mu}} \chi d \tau\right)^{n}\right] \sum_{p=0}^{\infty}\left[\frac{1}{p !}\left(i \int_{0}^{1} \frac{\delta_{l}}{\delta \rho_{4}} m \chi d \tau\right)^{p}\right] \\
& \times \exp \left[i \int_{0}^{1}(p \dot{x}+\pi \dot{\lambda}+v \dot{\chi}) d \tau\right] \exp \left(-i \int \rho^{n}\left(\tau_{1}\right) 2 \psi_{n}\left(\tau_{1}\right) d \tau_{1}\right) \\
& \times\left.\int \exp \left[\int_{0}^{1} \psi_{n} \dot{\psi}^{n} d \tau+\psi_{n}(1) \psi^{n}(0)\right]\right|_{\theta=0},
\end{aligned}
$$

para que seja possível aplicar cada uma das derivadas funcionais no termo

$$
\exp \left[\int\left(-i \int \rho^{n}\left(\tau_{1}\right) 2 \psi_{n}\left(\tau_{1}\right) d \tau_{1}\right)\right] .
$$

A seguir, mostro os resultados dessas derivações:

$$
\begin{aligned}
& \sum_{p=0}^{\infty}\left[\frac{1}{p !}\left(i \int_{0}^{1} \frac{\delta_{l}}{\delta \rho_{4}} m \chi d \tau\right)^{p}\right] \exp \left[\int\left(-i \int \rho^{n}\left(\tau_{1}\right) 2 \psi_{n}\left(\tau_{1}\right) d \tau_{1}\right)\right] \\
& =\exp \left(-i \int \rho^{n}\left(\tau_{1}\right) 2 \psi_{n}\left(\tau_{1}\right) d \tau_{1}\right) \exp \left(i \int_{0}^{1}-2 i m \psi_{4}(\tau) \chi d \tau\right), \\
& \sum_{n=0}^{\infty}\left[\frac{1}{n !}\left(-i \int_{0}^{1} \mathcal{P}_{\mu} \frac{\delta_{l}}{\delta \rho_{\mu}} \chi d \tau\right)^{n}\right] \exp \left[\int\left(-i \int \rho^{n}\left(\tau_{1}\right) 2 \psi_{n}\left(\tau_{1}\right) d \tau_{1}\right)\right] \\
& =\exp \left(-i \int \rho^{n}\left(\tau_{1}\right) 2 \psi_{n}\left(\tau_{1}\right) d \tau_{1}\right) \exp \left(i \int_{0}^{1} 2 i \mathcal{P}_{\mu} \psi_{\mu}(\tau) \chi d \tau\right)
\end{aligned}
$$

e

$$
\begin{aligned}
& \sum_{m=0}^{\infty}\left[\frac{1}{m !}\left(-\int_{0}^{1} \frac{g}{2} F_{\mu \nu} \frac{\delta_{l}}{\delta \rho_{\mu}} \frac{\delta_{l}}{\delta \rho_{\nu}} d \tau\right)^{m}\right] \exp \left(-i \int \rho^{n}\left(\tau_{1}\right) 2 \psi_{n}\left(\tau_{1}\right) d \tau_{1}\right) \\
& =\exp \left(-i \int \rho^{n}\left(\tau_{1}\right) 2 \psi_{n}\left(\tau_{1}\right) d \tau_{1}\right) \exp \left(i \int_{0}^{1} 2 i g F_{\mu \nu} \psi^{\mu}(\tau) \psi^{v}(\tau) d \tau\right)
\end{aligned}
$$

sendo $\frac{\delta_{l}}{\delta \rho_{\mu}}$ e $\chi$ ímpares. Os cálculos acima são similares aos cálculos feitos para provar 
a eq.6.5), presente no apêndice. É possível generalizar este resultado:

$$
\begin{aligned}
& \sum_{n=0}^{\infty}\left[\frac{1}{n !}\left(-i \int_{0}^{1} a \frac{\delta_{l}}{\delta \rho_{q}} d \tau\right)^{n}\right] \exp \left[\int\left(-i \int \rho^{n}\left(\tau_{1}\right) 2 \psi_{n}\left(\tau_{1}\right) d \tau_{1}\right)\right] \\
& =\exp \left(-i \int \rho^{n}\left(\tau_{1}\right) 2 \psi_{n}\left(\tau_{1}\right) d \tau_{1}\right) \exp \left(i \int_{0}^{1} a\left(-2 i \psi_{q}(\tau)\right) d \tau\right)
\end{aligned}
$$

onde a derivada parcial $\frac{\delta_{l}}{\delta \rho_{q}}$ deve ser deslocada para a direita e todas as outras variáveis e funções, representadas por $a$, devem ser deslocadas para a esquerda.

É possível também generalizar o resultado para o caso com mais de uma derivada funcional dentro da somatória:

$$
\begin{aligned}
& \sum_{n=0}^{\infty}\left[\frac{1}{n !}\left(-i \int_{0}^{1} a \frac{\delta_{l}}{\delta \rho_{q}} \cdots \frac{\delta_{l}}{\delta \rho_{r}} d \tau\right)^{n}\right] \exp \left[\int\left(-i \int \rho^{n}\left(\tau_{1}\right) 2 \psi_{n}\left(\tau_{1}\right) d \tau_{1}\right)\right] \\
& =\exp \left(-i \int \rho^{n}\left(\tau_{1}\right) 2 \psi_{n}\left(\tau_{1}\right) d \tau_{1}\right) \exp \left(i \int_{0}^{1} a\left(-2 i \psi_{q}(\tau)\right) \cdots\left(-2 i \psi_{r}(\tau)\right) d \tau\right) .
\end{aligned}
$$

Com a substituição dos resultados, encontramos que

$$
\begin{aligned}
& \tilde{S}^{C}\left(x_{\text {out }}, x_{\text {in }}\right)=\exp \left[i \Gamma^{n} \frac{\partial_{l}}{\partial \theta^{n}}\right] \int_{0}^{\infty} d \lambda_{0} \int_{0}^{\infty} d \chi_{0} \int D x D \lambda D \chi D p D \pi D \nu D \psi \\
& \times \exp i \int_{0}^{1}\left\{\left[-\lambda\left(m^{2}-\mathcal{P}^{2}\right)+2 i \lambda g F_{\mu \nu} \psi^{\mu} \psi^{\nu}-2 i\left(m \psi^{4}-\mathcal{P}_{\mu} \psi^{\mu}\right) \chi\right.\right. \\
& \left.\left.-i \psi_{n} \dot{\psi}^{n}+p \dot{x}+\pi \dot{\lambda}+v \dot{\chi}\right] d \tau+\psi_{n}(1) \psi^{n}(0)\right\}\left.\right|_{\theta=0},
\end{aligned}
$$

que pode ser reescrito como

$$
\begin{aligned}
\tilde{S}^{C}\left(x_{\text {out }}, x_{\text {in }}\right) & =\exp \left[i \Gamma^{n} \frac{\partial_{l}}{\partial \theta^{n}}\right] \int_{0}^{\infty} d \lambda_{0} \int_{0}^{\infty} d \chi_{0} \int D x D \lambda D \chi D p D \pi D v D \psi \\
& \left.\exp i \int_{0}^{1}\left\{[-\mathcal{H}(\lambda, \chi, x, p)+p \dot{x}+\pi \dot{\lambda}+v \dot{\chi}] d \tau+\psi_{n}(1) \psi^{n}(0)\right\}\right|_{\theta=0},
\end{aligned}
$$

onde

$\mathcal{H}(\lambda, \chi, x, p)=\lambda\left[m^{2}-\left(-p_{\mu}-g A_{\mu}\right)^{2}-2 i g \mathbb{F}_{\mu \nu} \psi^{\mu} \psi^{\nu}\right]+2 i\left(m \psi^{4}-\left(-p_{\mu}-g A_{\mu}\right) \psi^{\mu}\right) \chi+i \psi_{n} \dot{\psi}^{n}$

Para que a integral de trajetória esteja em sua forma Lagrangiana, é necessário, na equação acima, integrar sobre o momento $p$. Como no capítulo anterior, realizamos uma translação no momento, $p_{\mu} \rightarrow p_{\mu}+\tilde{p}_{\mu}$, onde $\tilde{p}_{\mu}$ é o momento clássico. 
O momento clássico é obtido a partir da equação

$$
\frac{\partial \mathcal{H}}{\partial p_{\alpha}}=\dot{x}^{\alpha}=-2 \lambda\left(p_{\alpha}+g A_{\alpha}\right)+2 i \psi^{\alpha} \chi
$$

Com isto, vemos que $\tilde{p}_{\mu}=-\frac{\dot{x}^{\mu}}{e}+2 i \frac{\psi^{\mu} \chi}{e}-g A_{\mu}$. Portanto, na eq.3.11 fazemos a seguinte substituição no momento, acompanhada da substituição em $\lambda$ :

$$
p_{\mu} \rightarrow p_{\mu}-\frac{\dot{x}^{\mu}}{e}+2 i \frac{\psi^{\mu} \chi}{e}-g A_{\mu}, \quad \lambda=e / 2
$$

Obtém-se, assim, que

$$
\begin{aligned}
& \tilde{S}^{C}\left(x_{\text {out }}, x_{\text {in }}\right)=\exp \left[i \Gamma^{n} \frac{\partial_{l}}{\partial \theta^{n}}\right] \int_{0}^{\infty} d \lambda_{0} \int_{0}^{\infty} d \chi_{0} \int D x D \lambda D \chi D p D \pi D \nu D \psi \\
& \times \mathcal{M}(e) \exp i \int_{0}^{1}\left\{\left[-\frac{\dot{x}^{2}}{2 e}-\frac{e}{2} m^{2}-g A_{\mu} \dot{x}+i e g \mathbb{F}_{\mu \nu} \psi^{\mu} \psi^{v}+2 i\left(\dot{x} \frac{\psi^{\mu}}{e}-m \psi^{4}\right) \chi+\right.\right. \\
& \left.\left.-i \psi_{n} \dot{\psi}^{n}+\pi \frac{\dot{e}}{2}+v \dot{\chi}\right] d \tau+\psi_{n}(1) \psi^{n}(0)\right\}\left.\right|_{\theta=0},
\end{aligned}
$$

onde foi usado que $\psi$ e $\chi$ são variáveis de Grassmann ímpares, e com isto, $\psi^{2}=\chi^{2}=0$.

A equação acima pode ser dividida em três partes: a primeira, é a medida de integração,

$$
\mathcal{M}(e)=\int D p \exp \left[\frac{i}{2} \int_{0}^{1} e p^{2} d \tau\right]
$$

a segunda, é a ação lagrangiana do sistema, invariante por transformação de calibre

$$
S=\int_{0}^{1}\left[-\frac{\dot{x}^{2}}{2 e}-\frac{e}{2} m^{2}-g A_{\mu} \dot{x}+i e g \mathbb{F}_{\mu \nu} \psi^{\mu} \psi^{\nu}+2 i\left(\dot{x} \frac{\psi^{\mu}}{e}-m \psi^{4}\right) \chi-i \psi_{n} \dot{\psi}^{n}\right] d \tau
$$

e a terceira, é o termo de fixação de calibre,

$$
S_{G F}=\int_{0}^{1}(\pi \dot{e}+v \dot{\chi}) d \tau
$$

que fixa $\dot{e}=0$ e $\dot{\chi}=0$.

Com isso finalizamos o capítulo 3. Neste capítulo obtivemos a ação pseudoclássica de um sistema muito semelhante ao sistema que trataremos no próximo capítulo, e por isso, parte dos resultados demonstrados aqui serão utilizados a seguir. 


\section{CAPÍTULO 4}

\section{Modelo pseudoclássico de uma partícula de Dirac que in- terage com o campo eletromagnético e interage, através da interação eletrofraca, com o campo de matéria}

Este é o principal capítulo deste trabalho, nele, obtemos novos resultados. Vamos obter o modelo pseudoclássico do sistema composto por férmions quirais que interagem com um campo de matéria, através da interação eletrofraca, e com o campo eletromagnético. Em seguida, na segunda parte do capítulo, vamos quantizar o novo modelo obtido.

Com os capítulos anteriores foi possível entender como usar o método de Fradkin e Gitman em sistemas fermiônicos, possibilitando a realização deste capítulo. Este sistema e o sistema do capítulo anterior possuem uma importante semelhança: os dois são descritos por equações de Dirac modificadas. Dessa forma, parte dos procedimentos que devemos aplicar aqui são os mesmos que aplicamos no sistema anterior. Como esses resultados já foram provados, ou melhor detalhados, no capítulo 3, vamos somente indicar que estamos usando eles novamente.

Para este sistema, partimos da seguinte equação retirada do artigo [56]:

$$
\left[\left(i \partial_{\mu}-g A_{\mu}(x)\right) \gamma^{\mu}-m-\gamma_{\mu}\left(V_{L}^{\mu}(x) P_{L}+V_{R}^{\mu}(x) P_{R}\right)\right] \Psi(x)=0,
$$

onde $V_{L, R}^{\mu}$ são os potenciais efetivos da interação eletrofraca entre o campo de matéria externo e os elétrons de mão direita e de mão esquerda, respectivamente. $P_{L, R}=(1 \mp$ $\left.\gamma^{5}\right) / 2$ são as projeções quirais, e $\gamma^{\mu}$, com $\mu=0,1,2,3$, e $\gamma^{5}=i \gamma^{0} \gamma^{1} \gamma^{2} \gamma^{3}$ são as matrizes de Dirac que satisfazem a relação $\left[\gamma^{\mu}, \gamma^{\nu}\right]_{+}=2 \eta^{\mu \nu}$.

Usaremos neste capítulo variáveis pares e ímpares da álgebra de Grassmann, cujas propriedades relevantes a este trabalho foram descritas no início do capítulo anterior. Usaremos também alguns resultados da álgebra de Berezin, eles serão apresentados no decorrer do capítulo. 


\subsection{Ação pseudoclássica}

Para obtermos a ação pseudoclássica reescrevemos a Eq. 4.1 da seguinte forma:

$$
\left\{\gamma^{v}\left[\hat{\mathcal{P}}_{v}-i \gamma^{5} K_{v}(x)\right]-m\right\} \Psi(x)=0
$$

onde

$$
\begin{aligned}
& \hat{\mathcal{P}}_{v}=i \partial_{v}-\mathbb{A}_{v}, \mathbb{A}_{v}(x)=g A_{v}(x)+V_{v}(x), \\
& V^{\mu}(x)=\left(V_{L}^{\mu}(x)+V_{R}^{\mu}(x)\right) / 2, K^{\mu}(x)=\left(V_{R}^{\mu}(x)-V_{L}^{\mu}(x)\right) / 2 .
\end{aligned}
$$

Utilizando que

$$
\gamma^{\rho} \gamma^{5}=\frac{i}{3 !} \eta^{\rho \alpha} \varepsilon_{\alpha \beta \mu \nu} \gamma^{\beta} \gamma^{\mu} \gamma^{v}
$$

onde $\varepsilon_{\alpha \beta \mu \nu}$ é um tensor completamente antissimétrico, e adotando a normalização $\varepsilon_{0123}=1$, a equação 4.2 torna-se:

$$
\left[\hat{\mathcal{P}}_{\nu} \gamma^{v}-m-\frac{i}{3 !} K^{\alpha}(x) \varepsilon_{\alpha \beta \mu \nu} \gamma^{\beta} \gamma^{\mu} \gamma^{v}\right] \Psi(x)=0 .
$$

A equação acima pode ser escrita em termos da função de Green causal, $S^{\mathrm{c}}(x, y)$, que satisfaz a equação não-homogênea abaixo:

$$
\left[\hat{\mathcal{P}}_{\nu} \gamma^{\nu}-m-\frac{i}{3 !} K^{\alpha}(x) \varepsilon_{\alpha \beta \mu \nu} \gamma^{\beta} \gamma^{\mu} \gamma^{\nu}\right] S^{\mathrm{c}}(x, y)=-\delta^{(4)}(x-y) .
$$

É conveniente multiplicarmos a equação acima por $\gamma^{5}$, da seguinte forma:

$$
\gamma^{5}\left[\hat{\mathcal{P}}_{\nu} \gamma^{\nu}-m-\frac{i}{3 !} K^{\alpha}(x) \varepsilon_{\alpha \beta \mu \nu} \gamma^{\beta} \gamma^{\mu}\left(-\gamma^{5} \gamma^{5}\right) \gamma^{\nu}\right] S^{\mathrm{c}}(x, y) \gamma^{5}=-\gamma^{5} \delta^{(4)}(x-y) \gamma^{5},
$$

para usarmos uma notação na qual $\tilde{S}^{\mathrm{c}}(x, y)=S^{\mathrm{c}}(x, y) \gamma^{5}$. Além disso, no lugar das matrizes $\gamma^{\mu}$ e $\gamma^{5}$ usaremos as matrizes $\Gamma^{n}, \operatorname{com} n=0,1,2,3,4$ :

$$
\Gamma^{\mu}=\gamma^{5} \gamma^{\mu}, \Gamma^{4}=\gamma^{5}, \mu=0,1,2,3
$$

Estas matrizes formam uma representação da álgebra de Clifford em cinco dimensões:

$$
\left[\Gamma^{n}, \Gamma^{m}\right]_{+}=2 \eta^{n m}, \eta^{n m}=\operatorname{diag}(1,-1,-1,-1,-1) .
$$

Com essas mudanças, obtemos a equação

$$
\left[\hat{\mathcal{P}}_{\nu} \Gamma^{v}-m \Gamma^{4}-\frac{i}{3 !} K^{\alpha}(x) \varepsilon_{\alpha \beta \mu \nu} \Gamma^{\beta} \Gamma^{\mu} \Gamma^{v}+i \epsilon\right] \tilde{S}^{\mathrm{c}}(x, y)=\delta^{(4)}(x-y) .
$$


Seguindo Schwinger [58], podemos escrever a função $\tilde{S}_{\alpha \beta}^{\mathrm{c}}(x, y)$ como um elemento de matriz do operador $\tilde{S}_{\alpha \beta}^{\mathrm{c}}$ :

$$
\tilde{S}_{\alpha \beta}^{\mathrm{c}}(x, y)=\left\langle x\left|\tilde{S}_{\alpha \beta}^{\mathrm{c}}\right| y\right\rangle .
$$

Os subíndices $\alpha, \beta$ são índices espinoriais e serão desconsiderados a partir daqui. $|x\rangle$ e $|y\rangle$ são os autovetores dos operadores posição $X^{\mu}$ e $Y^{\mu}$, respectivamente, e $P_{\mu}$ é o operador momento linear. Estes operadores e autovetores satisfazem as seguintes relações:

$$
\begin{aligned}
& X^{\mu}|x\rangle=x^{\mu}|x\rangle,\langle x \mid y\rangle=\delta^{4}(x-y), \int|x\rangle\langle x| d x=I, \\
& {\left[P_{\mu}, X^{\nu}\right]_{-}=-i \delta_{\mu}^{v}, P_{\mu}|p\rangle=p_{\mu}|p\rangle,\left\langle p \mid p^{\prime}\right\rangle=\delta^{4}\left(p-p^{\prime}\right),} \\
& \int|p\rangle\langle p| d p=I,\left\langle x\left|P_{\mu}\right| y\right\rangle=-i \partial_{\mu} \delta^{4}(x-y),\langle x \mid p\rangle=(2 \pi)^{-2} e^{i p x}, \\
& {\left[\Pi_{\mu}, \Pi_{\nu}\right]_{-}=-i \mathbb{F}_{\mu \nu}(X), \Pi_{\mu}=-P_{\mu}-\mathbb{A}_{\mu}(X) .}
\end{aligned}
$$

Como nos capítulos anteriores, encontramos o valor de $\tilde{S}^{c}$ com as transformações das funções em operadores. Dessa forma, podemos escrever a equação 4.7 como:

$$
\tilde{S}^{\mathrm{c}}=\left[\Pi_{\nu} \Gamma^{\nu}-m \Gamma^{4}-\frac{i}{3 !} K^{\alpha}(X) \varepsilon_{\alpha \beta \mu \nu} \Gamma^{\beta} \Gamma^{\mu} \Gamma^{\nu}\right]^{-1}
$$

O operador $\tilde{S}^{C}$ é um operador de Fermi, por possuir variáveis de Grassmann. As matrizes $\Gamma^{n}$ são consideradas operadores de Fermi. Gitman e Fradkin desenvolveram uma fórmula para escrever um operador de Fermi como a integral de uma exponencial:

$$
A^{-1}=\int_{0}^{\infty} d \lambda \int e^{i\left(\lambda\left(A^{2}+i \epsilon\right)+\chi A\right)} d \chi
$$

onde $A$ é um operador de Fermi ímpar, $\lambda$ é uma variável de Grassmann par e $\chi$ uma variável de Grassmann ímpar que, por definição, anticomuta com $A$ - veja a Ref. [1]. Mesmo que uma variável de Grassmann elevada ao quadrado seja igual a zero, podemos elevar $A$ ao quadrado já que ela é uma combinação de operadores escritos com as variáveis de Grassmann e essa combinação elevada ao quadrado não é necessariamente zero. Podemos resumir o que foi feito até agora como:

$$
\begin{aligned}
\tilde{S}^{\mathrm{c}}\left(x_{\text {out }}, x_{\text {in }}\right) & =\left\langle x_{\text {out }}\left|\tilde{S}^{\mathrm{c}}\right| x_{\text {in }}\right\rangle \\
& =\left\langle x_{\text {out }}\left|\left[\Pi_{\nu} \Gamma^{v}-m \Gamma^{4}-\frac{i}{3 !} K^{\alpha}(X) \varepsilon_{\alpha \beta \mu \nu} \Gamma^{\beta} \Gamma^{\mu} \Gamma^{v}\right]^{-1}\right| x_{\text {in }}\right\rangle \\
& =\left\langle x_{\text {out }}\left|\int_{0}^{\infty} d \lambda \int e^{-i \mathcal{H}(\lambda, \chi, X, P)} d \chi\right| x_{\text {in }}\right\rangle,
\end{aligned}
$$


onde

$$
-i \mathcal{H}(\lambda, \chi, X, P)=i\left[\lambda\left(A^{2}+i \epsilon\right)+\chi A\right] .
$$

O cálculo de $A^{2}$ nos fornece

$$
A^{2}=\left(\Pi_{v} \Gamma^{\mu}-\Gamma^{4} m\right)^{2}-\Pi_{v} \Gamma^{\mu} c-c \Pi_{v} \Gamma^{\mu}+m\left(c \Gamma^{5}+\Gamma^{5} c\right)+c^{2},
$$

onde $c=\frac{i}{3 !} K^{\alpha}(X) \varepsilon_{\alpha \beta \mu \nu} \Gamma^{\beta} \Gamma^{\mu} \Gamma^{v}$, e

$$
\begin{aligned}
& \left(\Pi_{\mu} \Gamma^{\mu}-\Gamma^{4} m\right)^{2}=\Pi^{2}-m^{2}-\frac{i}{2} \mathbb{F}_{\alpha \beta} \Gamma^{\alpha} \Gamma^{\beta}, \\
& -\Pi_{\mu} \Gamma^{\mu} c-c \Pi_{\mu} \Gamma^{\mu}=\partial_{\mu} K^{\mu} \Gamma^{0} \Gamma^{1} \Gamma^{2} \Gamma^{3}+\frac{i}{2} \epsilon^{\alpha \beta \mu \nu}\left[\Pi_{\alpha}, K_{\beta}\right]_{+} \Gamma_{\mu} \Gamma_{v}, \\
& c \Gamma^{5}+\Gamma^{5} c=0, \\
& c^{2}=-K^{2} .
\end{aligned}
$$

Portanto,

$$
\begin{aligned}
A^{2}= & \Pi^{2}-m^{2}-\frac{i}{2} \mathbb{F}_{\alpha \beta} \Gamma^{\alpha} \Gamma^{\beta}-K^{2}+\partial_{\mu} K^{\mu} \Gamma^{0} \Gamma^{1} \Gamma^{2} \Gamma^{3}, \\
& +\frac{i}{2} \varepsilon^{\alpha \beta \mu v}\left[\Pi_{\alpha}, K_{\beta}\right]_{+} \Gamma_{\mu} \Gamma_{v}, \mathbb{F}_{\alpha \beta}=\partial_{\alpha} \mathbb{A}_{\beta}-\partial_{\beta} \mathbb{A} .
\end{aligned}
$$

Com isso, obtemos que

$$
\begin{aligned}
\hat{\mathcal{H}}(\lambda, \chi, X, P)= & \lambda\left\{m^{2}-\Pi^{2}+\frac{i}{2} \mathbb{F}_{\alpha \beta} \Gamma^{\alpha} \Gamma^{\beta}+K^{2}-\frac{i}{2}\left[\Pi_{\alpha}, K_{\beta}\right]_{+} \varepsilon^{\alpha \beta \mu \nu} \Gamma_{\mu} \Gamma_{v}+\right. \\
& \left.-\partial_{\mu} K^{\mu} \Gamma^{0} \Gamma^{1} \Gamma^{2} \Gamma^{3}\right\}+\left(\Pi_{v} \Gamma^{v}-m \Gamma^{4}-\frac{i}{3 !} K^{\alpha} \varepsilon_{\alpha \beta \mu \nu} \Gamma^{\beta} \Gamma^{\mu} \Gamma^{v}\right) \chi .
\end{aligned}
$$

Utilizando o resultado acima, junto com o resultado já provado no capítulo 3 que nos diz que

$$
\tilde{S}^{c}\left(x_{\text {out }}, x_{\text {in }}\right)=\int_{0}^{\infty} d \lambda \int\left\langle x_{\text {out }}\left|e^{-i \hat{\mathcal{H}}(\lambda, \chi, X, P)}\right| x_{\text {in }}\right\rangle d \chi
$$

pode ser escrito como a integral de trajetória

$$
\begin{aligned}
\tilde{S}^{c}\left(x_{\text {out }}, x_{\text {in }}\right)= & \int_{0}^{\infty} d \lambda_{0} d \chi_{0} \mathcal{D} x \mathcal{D} p \mathcal{D} \lambda \mathcal{D} \pi \mathcal{D} \chi \mathcal{D} v \\
& \times \int \exp \left\{i \int_{0}^{1}[-\mathcal{H}(\lambda, \chi, x, p)+p \dot{x}+\pi \dot{\lambda}+v \dot{\chi}] d \tau\right\},
\end{aligned}
$$

onde $\mathcal{H}(\lambda, \chi, x, p)$ é o símbolo de Weyl do operador $\hat{\mathcal{H}}(\lambda, \chi, X, P)$, eq. 4.11). Substituindo 
o símbolo de Weyl na equação acima obtemos:

$$
\begin{aligned}
& \tilde{S}^{\mathrm{c}}\left(x_{\text {out }}, x_{\text {in }}\right)=\int_{0}^{\infty} d \lambda_{0} d \chi_{0} \mathcal{D} x \mathcal{D} p \mathcal{D} \lambda \mathcal{D} \pi \mathcal{D} \chi \mathcal{D} v \\
& \times \int \exp \left\{i \int _ { 0 } ^ { 1 } \left[\lambda \left(\mathcal{P}^{2}-m^{2}-\frac{i}{2} \mathbb{F}_{\alpha \beta} \Gamma^{\alpha} \Gamma^{\beta}-K^{2}+i \varepsilon^{\alpha \beta \mu \nu} \mathcal{P}_{\alpha} K_{\beta} \Gamma_{\mu} \Gamma_{v}\right.\right.\right. \\
& \left.+\partial_{\mu} K^{\mu} \Gamma^{0} \Gamma^{1} \Gamma^{2} \Gamma^{3}\right)+\left(m \Gamma^{4}-\mathcal{P}_{\nu} \Gamma^{v}+\frac{i}{3 !} K^{\alpha} \varepsilon_{\alpha \beta \mu \nu} \Gamma^{\beta} \Gamma^{\mu} \Gamma^{v}\right) \chi \\
& +p \dot{x}+\pi \dot{\lambda}+v \dot{\chi}] d \tau\}
\end{aligned}
$$

onde $\mathcal{P}_{v}=-p_{v}-\mathbb{A}_{v}(x) ; x(\tau), p(\tau), \lambda(\tau)$ e $\pi(\tau)$ são trajetórias pares; e $\chi(\tau), v(\tau)$ são trajetórias ímpares. Todas as trajetórias obedecem os limites de integração $x(0)=x_{\text {in }}$, $x(1)=x_{\text {out }}$ e $\lambda(0)=\lambda_{0}, \chi(0)=\chi_{0}$.

Como no sistema anterior, a equação acima depende das matrizes $\Gamma^{\alpha}$, para que isso não ocorra, usamos que $\Gamma^{\alpha}$ pode ser escrita como:

$$
\frac{\delta_{l}}{\delta \rho_{\alpha}}\left[\left.T\left(\exp \int_{0}^{1} \rho_{n}(\tau) \Gamma^{n} d \tau\right)\right|_{\rho=0}\right]=\Gamma^{\alpha}\left[\left.T\left(\exp \int_{0}^{1} \rho_{n}(\tau) \Gamma^{n} d \tau\right)\right|_{\rho=0}\right]=\Gamma^{\alpha},
$$

onde $T\left(\exp \int_{0}^{1} \rho_{n}(\tau) \Gamma^{n} d \tau\right)$ é uma exponencial ordenada no tempo, esse ordenamento é necessário porque $\rho_{n}(\tau) \Gamma^{n}$ não comuta com $\rho_{n}\left(\tau^{\prime}\right) \Gamma^{n}$ quando $\tau \neq \tau^{\prime}$. Escrever $\Gamma^{\alpha}$ nesta forma foi pensada por Fradkin e Gitman. Aplicando este resultado na (4.12) resulta em:

$$
\begin{aligned}
& \tilde{S}^{\mathrm{c}}\left(x_{\text {out }}, x_{\mathrm{in}}\right)=\int_{0}^{\infty} d \lambda_{0} d \chi_{0} \mathcal{D} \times \mathcal{D} p \mathcal{D} \lambda \mathcal{D} \pi \mathcal{D} \chi \mathcal{D} v \\
& \int \exp \left\{i \int _ { 0 } ^ { 1 } \left[\lambda \left(\mathcal{P}^{2}-m^{2}-\frac{i}{2} \mathbb{F}_{\alpha \beta} \frac{\delta_{l}}{\delta \rho_{\alpha}} \frac{\delta_{l}}{\delta \rho_{\beta}}-K^{2}\right.\right.\right. \\
& \left.+i \varepsilon^{\alpha \beta \mu \nu} \mathcal{P}_{\alpha} K_{\beta} \frac{\delta_{l}}{\delta \rho_{\mu}} \frac{\delta_{l}}{\delta \rho_{v}}+\partial_{\mu} K^{\mu} \frac{\delta_{l}}{\delta \rho_{0}} \frac{\delta_{l}}{\delta \rho_{1}} \frac{\delta_{l}}{\delta \rho_{2}} \frac{\delta_{l}}{\delta \rho_{3}}\right)+\left(m \frac{\delta_{l}}{\delta \rho_{5}}-\mathcal{P}_{\nu} \frac{\delta_{l}}{\delta \rho_{\nu}}\right. \\
& \left.\left.\left.+\frac{i}{3 !} K^{\alpha} \varepsilon_{\alpha \beta \mu \nu} \frac{\delta_{l}}{\delta \rho_{\beta}} \frac{\delta_{l}}{\delta \rho_{\mu}} \frac{\delta_{l}}{\delta \rho_{v}}\right) \chi+p \dot{x}+\pi \dot{\lambda}+v \dot{\chi}\right] d \tau\right\} \\
& \times\left.\operatorname{Texp} \int_{0}^{1} \rho_{n}(\tau) \Gamma^{n} d \tau\right|_{\rho=0},
\end{aligned}
$$

onde introduzimos cinco fontes ímpares $\rho_{n}(\tau)$, que, por definição, anticomutam com as matrizes $\Gamma^{n}$. 
Utilizando o resultado provado no apêndice, que nos diz que

$$
\begin{aligned}
T \exp \left[\int_{0}^{1} \rho_{n}(\tau) \Gamma^{n} d \tau\right]= & \exp \left[i \Gamma^{n} \frac{\partial_{l}}{\partial \theta^{n}}\right] \\
& \times\left.\int D \psi \exp \left[\left(\int_{0}^{1} \psi_{n} \dot{\psi}^{n}-2 i \rho_{n} \psi^{n}\right) d \tau+\psi_{n}(1) \psi^{n}(0)\right]\right|_{\theta=0},
\end{aligned}
$$

onde $\psi^{n}$ são variáveis ímpares, obtemos que

$$
\begin{aligned}
& \tilde{S}^{\mathrm{c}}\left(x_{\text {out }}, x_{\text {in }}\right)=\exp \left(i \Gamma^{n} \frac{\partial_{l}}{\partial \theta^{n}}\right) \int_{0}^{\infty} d \lambda_{0} d \chi_{0} D x D p D \lambda D \pi D \chi D \nu \\
& \times \int_{\psi(0)+\psi(1)=\theta} \mathcal{D} \psi \int \exp \left\{i \int_{0}^{1}[p \dot{x}-\mathcal{H}(\lambda, \chi, x, p)+\pi \dot{\lambda}+v \dot{\chi}] d \tau\right\} \\
& \times\left.\exp \left(-i \int \Gamma^{n}\left(\tau_{1}\right) 2 \psi_{n}\left(\tau_{1}\right) d \tau_{1}\right) \exp \left[\int_{0}^{1} \psi_{n} \dot{\psi}^{n} d \tau+\psi_{n}(1) \psi^{n}(0)\right]\right|_{\theta=0} .
\end{aligned}
$$

Tal como no capítulo anterior, os termos $\exp \left[i \int_{0}^{1}[p \dot{x}-\mathcal{H}(\lambda, \chi, x, p)+\pi \dot{\lambda}+\nu \dot{\chi}] d \tau\right]$ devem ser expandidos em séries de Taylor. Com isso, as derivadas $\frac{\delta}{\delta \rho_{\mu}}$ podem ser aplicados na exponencial $\exp \left(-2 i \int \rho^{n} \psi_{n} d \tau_{1}\right)$, o que nos fornece a seguinte representação da função de Green:

$$
\begin{aligned}
& \tilde{S}^{\mathrm{c}}\left(x_{\mathrm{out}}, x_{\mathrm{in}}\right)=\exp \left(i \Gamma^{n} \frac{\partial_{l}}{\partial \theta^{n}}\right) \int_{0}^{\infty} d \lambda_{0} \int d \chi_{0} \mathcal{D} x \mathcal{D} p \mathcal{D} \lambda \mathcal{D} \pi \mathcal{D} \chi \mathcal{D} \nu \mathcal{D} \psi \\
& \times \int_{\psi(0)+\psi(1)=\theta} \mathcal{D} \psi \exp \left\{i \int _ { 0 } ^ { 1 } \left[\lambda \left(\mathcal{P}^{2}-m^{2}+2 i \mathbb{F}_{\alpha \beta} \psi^{\alpha} \psi^{\beta}-K^{2}\right.\right.\right. \\
& \left.-4 i \varepsilon^{\alpha \beta \mu \nu} \mathcal{P}_{\alpha} K_{\beta} \psi_{\mu} \psi_{v}+16 \partial_{\mu} K^{\mu} \psi^{0} \psi^{1} \psi^{2} \psi^{3}\right)+2 i\left(\mathcal{P}_{\alpha} \psi^{\alpha}-m \psi^{4}+\frac{2 i}{3} K^{\alpha} \varepsilon_{\alpha \beta \mu \nu} \psi^{\beta} \psi^{\mu} \psi^{v}\right) \chi \\
& \left.\left.-i \psi_{n} \dot{\psi}^{n}+p \dot{x}+\pi \dot{\lambda}+v \dot{\chi}\right] d \tau+\psi_{n}(1) \psi^{n}(0)\right\}\left.\right|_{\theta=0} .
\end{aligned}
$$

A Hamiltoniana $\mathcal{H}(\lambda, \chi, x, y)$ é extraída da equação acima:

$$
\begin{aligned}
\mathcal{H}(\lambda, \chi, x, y) & =-\lambda\left(\mathcal{P}^{2}-m^{2}+2 i F_{\alpha \beta} \psi^{\alpha} \psi^{\beta}+16 \partial_{\mu} K^{\mu} \psi^{0} \psi^{1} \psi^{2} \psi^{3}-4 i \epsilon^{\alpha \beta \mu \nu} \mathcal{P}_{\alpha} K_{\beta} \psi_{\mu} \psi_{v}-K^{2}\right) \\
& +2 i\left(\mathcal{P}_{\mu} \psi^{\mu}-\psi^{4} m+\frac{2 i}{3} K^{\alpha} \epsilon_{\alpha \beta \mu \nu} \psi^{\beta} \psi^{\mu} \psi^{\nu}\right) \chi
\end{aligned}
$$

Para que a integral de trajetória esteja em sua forma Lagrangiana, é necessário, na equação acima, integrar sobre o momento $p$. Como no capítulo anterior, realizamos uma translação no momento, $p_{\mu} \rightarrow p_{\mu}+\tilde{p}_{\mu}$, onde $\tilde{p}_{\mu}$ é o momento clássico. 
O momento clássico é obtido a partir da equação $p_{\alpha}^{\prime}$, a partir de $\frac{\partial H}{\partial p_{\zeta}}=\dot{x}^{\zeta}$ :

$$
\frac{\partial H}{\partial p_{\zeta}}=\frac{\partial}{\partial p_{\zeta}}\left[-\lambda\left(\mathcal{P}^{2}-4 i \epsilon^{\alpha \beta \mu \nu} \mathcal{P}_{\alpha} K_{\beta} \psi_{\mu} \psi_{\nu}\right)+2 i \mathcal{P}_{\mu} \psi^{\mu} \chi\right]
$$

Resolvendo a derivada acima, obtemos que

$$
p^{\prime \zeta}=\frac{-\dot{x}^{\zeta}-4 \lambda i \epsilon^{\zeta \beta \mu v} K_{\beta} \psi_{\mu} \psi_{v}+2 i \psi^{\zeta} \chi}{2 \lambda}-\mathbb{A}^{\zeta}
$$

Em seguida, fazemos a substituição do momento $p_{\alpha}$, presente em $\mathcal{P}_{\alpha}=-p_{\alpha}-\mathbb{A}_{\alpha}(x)$, por $p_{\alpha} \rightarrow p_{\alpha}+p_{\alpha}^{\prime}$, acompanhada de substituições em $\chi$ e $e$ :

$$
p_{\alpha} \rightarrow p_{\alpha}+p_{\alpha}^{\prime}, \quad \chi \rightarrow \chi / 2, \quad \lambda \rightarrow e / 2
$$

Assim, obtemos que

$$
\begin{aligned}
& \tilde{S}^{\mathrm{c}}\left(x_{\text {out }}, x_{\text {in }}\right)=\exp \left(i \Gamma^{n} \frac{\partial_{l}}{\partial \theta^{n}}\right) \int_{0}^{\infty} d e_{0} \int_{0}^{\infty} d \chi_{0} \int D e D \chi D x D \pi D \nu \mathcal{D} \psi \\
& \times \mathcal{M}(e) \exp \left\{i \int _ { 0 } ^ { 1 } \left[-\frac{\dot{x}^{2}}{2 e}-\frac{e}{2}\left(M^{2}-2 i \mathbb{F}_{\alpha \beta} \psi^{\alpha} \psi^{\beta}\right)-\dot{x}^{\alpha}\left(\mathbb{A}_{\alpha}-d_{\alpha}\right)\right.\right. \\
& \left.\left.+i\left(\frac{\dot{x}_{\alpha} \psi^{\alpha}}{e}-m \psi^{4}-\frac{2}{3} \psi^{\alpha} d_{\alpha}\right) \chi-i \psi_{n} \dot{\psi}^{n}+\pi \dot{e}+v \dot{\chi}\right] d \tau+\psi_{n}(1) \psi^{n}(0)\right\}\left.\right|_{\theta=0},
\end{aligned}
$$

onde

$$
M^{2}=m^{2}+K^{2}-16 \partial_{\mu} K^{\mu} \psi^{0} \psi^{1} \psi^{2} \psi^{3}, \quad d_{\alpha}=-2 i \varepsilon_{\alpha \beta \mu \nu} K^{\beta} \psi^{\mu} \psi^{\nu} .
$$

Esta é a forma final da integral de trajetória do nosso sistema. Ela pode ser dividida em três partes, a primeira delas é a medida de integração $\mathcal{M}(e)$ :

$$
\mathcal{M}(e)=\int \mathcal{D} p \exp \left[\frac{i}{2} \int_{0}^{1} e p^{2} d \tau\right]
$$

A segunda é a ação lagrangiana

$$
\begin{aligned}
S= & \int_{0}^{1}\left[-\frac{\dot{x}^{2}}{2 e}-\frac{e}{2}\left(M^{2}-2 i \mathbb{F}_{\alpha \beta} \psi^{\alpha} \psi^{\beta}\right)-\dot{x}^{\alpha}\left(\mathbb{A}_{\alpha}-d_{\alpha}\right)\right. \\
& \left.+i\left(\frac{\dot{x}_{\alpha} \psi^{\alpha}}{e}-m \psi^{4}-\frac{2}{3} \psi^{\alpha} d_{\alpha}\right) \chi-i \psi_{n} \dot{\psi}^{n}\right] d \tau,
\end{aligned}
$$

invariante às seguintes transformações de calibre, que representam uma reparametrização no tempo:

$$
\delta x^{\alpha}=\dot{x}^{\alpha} \xi, \delta e=\frac{d}{d \tau}(e \xi), \delta \chi=\frac{d}{d \tau}(\chi \xi), \delta \psi^{n}=\dot{\psi}^{n} \xi
$$


sendo $\xi$ um parâmetro par.

E por último, o termo de fixação de calibre

$$
S_{\mathrm{GF}}=\int_{0}^{1}(\pi \dot{e}+v \dot{\chi}) d \tau,
$$

que fixa $\dot{e}=0$ e $\dot{\chi}=0$.

Finalizamos, assim, esta seção com a obtenção da ação peseudoclássica do sistema estudado. Na próxima seção iremos quantizar o modelo aqui obtido.

\subsection{Quantização da ação pseudoclássica}

Nesta seção partimos da ação lagrangiana da equação (4.15) e obtemos a Hamiltoniana correspondente. A Lagrangiana é singular e, em decorrência disso, o sistema possui vínculos que são obtidos através do algoritmo de Dirac-Bergmann. O sistema será quantizado com a quantização de Dirac, para isso, devemos obter os vínculos da teoria e classificá-los em vínculos de primeira e segunda classe; a referência [57] foi usada. No final do processo, obtivemos um sistema quântico que equivale ao nosso sistema inicial: o sistema descrito pela eq. (4.1).

\section{Equações de Euler-Lagrange}

$\mathrm{Na}$ álgebra de Berezin, as equações de movimento da teoria obedecem a seguinte equação:

$$
\frac{\delta_{r} S}{\delta q^{i}}=\frac{\partial_{r} L}{\partial q^{i}}-\frac{d}{d t} \frac{\partial_{r} L}{\partial \dot{q}^{i}}=0,
$$

onde $q^{i}$ são as coordenadas generalizadas do sistema, $q=\left(x, e, \chi, \psi^{n}\right)$.

As equações de movimento da ação (4.15) são da seguinte forma:

$$
\begin{aligned}
& \frac{\delta S}{\delta e}=\frac{1}{e^{2}}\left(\frac{\dot{x}^{2}}{2}-i \dot{x}_{\alpha} \psi^{\alpha} \chi\right)-\frac{1}{2}\left(M^{2}-2 i \mathbb{F}_{\alpha \beta} \psi^{\alpha} \psi^{\beta}\right)=0, \\
& \frac{\delta_{r} S}{\delta \chi}=\frac{\dot{x}_{\alpha} \psi^{\alpha}}{e}-m \psi^{4}-\frac{2}{3} \psi^{\alpha} d_{\alpha}=0, \\
& \frac{\delta_{r} S}{\delta \psi^{\alpha}}=2 i \dot{\psi}_{\alpha}+2 e\left(i \mathbb{F}_{\beta \alpha} \psi^{\beta}+\frac{2}{3} \partial_{\sigma} K^{\sigma} \varepsilon_{\beta \mu \nu \alpha} \psi^{\beta} \psi^{\mu} \psi^{v}\right)+2 i d_{\alpha} \chi- \\
& i \frac{\dot{x}_{\alpha}}{e} \chi-4 i \varepsilon_{\mu \nu \beta \alpha} \dot{x}^{\mu} K^{v} \psi^{\beta}=0, \\
& \frac{\delta_{r} S}{\delta \psi^{4}}=-2 i \dot{\psi}^{4}+i m \chi=0, \\
& \frac{\delta S}{\delta x^{\alpha}}=\frac{d}{d \tau}\left(\frac{\dot{x}_{\alpha}-i \psi_{\alpha} \chi}{e}+\mathbb{A}_{\alpha}-d_{\alpha}\right)+8 e\left(\partial_{\alpha} \partial_{\mu} K^{\mu}\right) \psi^{0} \psi^{1} \psi^{2} \psi^{3}+i e \mathbb{F}_{\mu v, \alpha} \psi_{\mu} \psi_{v^{-}}- \\
& \dot{x}^{\mu}\left(\partial_{\alpha} \mathbb{A}_{\mu}\right)+\dot{x}^{\mu}\left(\partial_{\alpha} d_{\mu}\right)-e K^{\mu}\left(\partial_{\alpha} K_{\mu}\right)+\frac{2 i}{3} \chi \psi^{\mu}\left(\partial_{\alpha} d_{\mu}\right)=0 .
\end{aligned}
$$




\section{Algoritmo de Dirac-Bergmann - Hamiltoniana e vínculos primários}

O primeiro passo do Algoritmo de Dirac-Bergmann é obter a Hamiltoniana do sistema a partir da Lagrangiana da equação (4.15). Escrevemos $H$ como

$$
H=\left.\left(\frac{\partial_{r} L}{\partial \dot{q}} \dot{q}-L\right)\right|_{\frac{\partial_{r} L}{\partial \dot{q}}=P},
$$

onde $q=\left(x, e, \chi, \psi^{n}\right)$ são as coordenadas generalizadas e $P=\left(p, P_{e}, P_{\chi}, P_{n}\right)$ são os momentos conjugados.

Os momentos conjugados podem ser calculados através da equação $P_{i}=\frac{\partial_{r} L}{\partial \dot{q}_{i}}$ :

$$
\begin{gathered}
p_{\alpha}=\frac{\partial L}{\partial \dot{x}^{\alpha}}=-\frac{1}{e}\left(\dot{x}_{\alpha}-i \psi_{\alpha} \chi\right)-A_{\alpha}-d_{\alpha}, \\
P_{e}=\frac{\partial L}{\partial \dot{e}}=0, \quad P_{\chi}=\frac{\partial L}{\partial \dot{\chi}}, \quad P_{n}=\frac{\partial_{r} L}{\partial \dot{\psi}^{n}}=-i \psi^{n} .
\end{gathered}
$$

Das equações acima, obtemos que

$$
\dot{x}_{\alpha}=-e p_{\alpha}-e \mathbb{A}_{\alpha}-e d_{\alpha}+i \psi_{\alpha} \chi
$$

e substituímos em $H$,

$$
H=-\frac{e}{2}\left(\mathcal{P}^{2}+2 \mathcal{P}^{\alpha} d_{\alpha}+2 i \mathbb{F}_{\alpha \beta} \psi^{\alpha} \psi^{\beta}-M^{2}\right)-i\left(\mathcal{P}_{\alpha} \psi^{\alpha}-m \psi^{4}+\frac{1}{3} \psi^{\alpha} d_{\alpha}\right) \chi
$$

onde $\mathcal{P}_{\alpha}=-p_{\alpha}-\mathbb{A}_{\alpha}$.

Como não foi possível obter as velocidades generalizadas $\dot{e}, \dot{\chi}$ e $\dot{\psi}^{n}$ em função dos momentos conjugados, este sistema possui vínculos primários, aqui representados por $\Phi_{A}^{(1)}=0$. Tais vínculos primários podem ser obtidos a partir das equações em 4.18 :

$$
\Phi_{A}^{(1)}=\left\{\begin{array}{l}
\Phi_{1}^{(1)}=P_{\chi}, \\
\Phi_{2}^{(1)}=P_{e}, \\
\Phi_{3 n}^{(1)}=P_{n}+i \psi_{n} .
\end{array}\right.
$$

A Hamiltoniana com o acréscimo dos vínculos é chamada de Hamiltoniana modiicada e será representada por $H^{(1)}$. Os vínculos são incorporados a esta Hamiltoniana através dos multiplicadores de Lagrange $\lambda_{A}$.

Usaremos a notação do livro [57]:

$$
\begin{aligned}
H^{(1)} & =H+\sum_{i} \lambda_{A} \Phi_{A}^{(1)} \\
& =H+\lambda_{1} P_{\chi}+\lambda_{2} P_{e}+\lambda_{3, n}\left(P_{n}+i \psi_{n}\right) .
\end{aligned}
$$




\section{Algoritmo de Dirac-Bergmann - vínculos secundários e multiplicadores de Lagrange}

Os vínculos secundários e os multiplicadores de Lagrange são obtidos com o uso da condição de consistência, ela nos diz que todos os vínculos do sistema possuem a derivada total em relação ao tempo igual a zero: $\dot{\Phi}=0$.

Uma forma muito útil de calcular a derivada total de uma função $F\left(q_{i}, p^{i}\right)$, dependente das coordenadas e dos momentos generalizados do sistema, é usando a seguinte equação:

$$
\dot{F}=\left\{F, H^{(1)}\right\}=\{F, H\}+\sum_{i} \lambda_{i}\left\{F, \Phi_{i}^{(1)}\right\}
$$

onde $H^{(1)}$ é a Hamiltoniana modificada e $\left\{F, H^{(1)}\right\}$ é o parênteses de Poisson entre $F$ e $H^{(1)}$. O parênteses de Poisson na álgebra de Berezin, das funções $F\left(q_{i}, p^{i}\right)$ e $G\left(q_{i}, p^{i}\right)$ é definido como:

$$
\{F, G\}=\frac{\partial F}{\partial q^{i}} \frac{\partial G}{\partial p_{i}}-(-1)^{P_{F} P_{G}} \frac{\partial G}{\partial q^{i}} \frac{\partial F}{\partial p_{i}}
$$

onde $P_{F}$ depende da paridade da função $F\left(q_{i}, p^{i}\right)$. Se $F\left(q_{i}, p^{i}\right)$ for par, $P_{F}=0$. Se $F\left(q_{i}, p^{i}\right)$ for ímpar, $P_{F}=1$. O mesmo vale para $P_{G}$, que depende da paridade de $G\left(q_{i}, p^{i}\right)$.

Para as coordenadas e momentos do nosso sistema, o parênteses de Poisson é da forma:

$$
\begin{aligned}
\{F, G\} & =\frac{\partial F}{\partial x^{\mu}} \frac{\partial G}{\partial p_{\mu}}-(-1)^{P_{F} P_{G}} \frac{\partial G}{\partial x^{\mu}} \frac{\partial F}{\partial p_{\mu}}+\frac{\partial F}{\partial e} \frac{\partial G}{\partial P_{e}}-(-1)^{P_{F}} P_{G} \frac{\partial G}{\partial e} \frac{\partial F}{\partial P_{e}} \\
& +\frac{\partial_{r} F}{\partial \psi^{n}} \frac{\partial_{l} G}{\partial P_{n}}-(-1)^{P_{F} P_{G}} \frac{\partial_{r} G}{\partial \psi^{n}} \frac{\partial_{l} F}{\partial P_{n}}+\frac{\partial_{r} F}{\partial \chi} \frac{\partial_{l} G}{\partial P_{\chi}}-(-1)^{P_{F} P_{G}} \frac{\partial_{r} G}{\partial \chi} \frac{\partial_{l} F}{\partial P_{\chi}},
\end{aligned}
$$

vale lembrar que $x, p$,e são variáveis pares, $\chi, \psi^{n}$ são variáveis ímpares e a Hamiltoniana $H^{(1)}$ é par.

Ao aplicarmos a condição de consistência em um vínculo, três resultados são possíveis:

1. A condição de consistência nos fornece $0=0$, e não obtemos novas informações sobre o sistema;

2. A condição de consistência nos fornece algum dos multiplicadores de Lagrange;

3. A condição de consistência resulta em uma função dependente das coordenadas e dos momentos generalizados $q_{i}$ e $p^{i}$. Essa função é um novo vínculo da teoria e devemos aplicar a condição de consistência em todo novo vínculo obtido.

Vamos primeiro aplicar a condição de consistência nos vínculos primários, eq. (4.19): 


$$
\begin{gathered}
\dot{\Phi}_{1}^{(1)}=\left\{P_{\chi}, H^{(1)}\right\}=-\frac{\partial_{r} H^{(1)}}{\partial \chi}=-i\left(\mathcal{P}_{\alpha} \psi^{\alpha}-m \psi^{4}+\frac{1}{3} \psi^{\alpha} d_{\alpha}\right)=0, \\
\dot{\Phi}_{2}^{(1)}=\left\{P_{e}, H^{(1)}\right\}=-\frac{\partial_{r} H^{(1)}}{\partial e}=-\frac{e}{2}\left(\mathcal{P}^{2}-2 \mathcal{P}^{\alpha} d_{\alpha}+2 i \mathbb{F}_{\alpha \beta} \psi^{\alpha} \psi^{\beta}-M^{2}\right)=0,
\end{gathered}
$$

o que nos fornece os vínculos secundários:

$$
\begin{aligned}
& \Phi_{1}^{(2)}=\mathcal{P}_{\alpha} \psi^{\alpha}-m \psi^{4}+\frac{1}{3} \psi^{\alpha} d_{\alpha}=0, \\
& \Phi_{2}^{(2)}=\mathcal{P}^{2}+2 \mathcal{P}^{\alpha} d_{\alpha}+2 i \mathbb{F}_{\alpha \beta} \psi^{\alpha} \psi^{\beta}-M^{2}=0 .
\end{aligned}
$$

Podemos ver que a Hamiltoniana pode ser escrita em termos dos vínculos $\Phi_{1}^{(2)} \mathrm{e}$ $\Phi_{2}^{(2)}$ :

$$
H=i \chi \Phi_{1}^{(2)}-\frac{e}{2} \Phi_{2}^{(2)}
$$

tal como deve ser no caso de uma teoria invariante por reparametrização. Usaremos a Hamiltoniana nesta forma para facilitar as contas.

Aplicando a condição de consistência em $\Phi_{3, n}^{(1)}$ :

$$
\dot{\Phi}_{3, n}^{(1)}=\left\{P_{n}+i \psi_{n}, H^{(1)}\right\}=i \frac{\partial_{l} H^{(1)}}{\partial P_{n}}-\frac{\partial_{r} H^{(1)}}{\partial \psi^{n}}
$$

obtemos o multiplicador de Lagrange $\lambda_{3, n}$ :

$$
\lambda_{3 n}=-\frac{i e}{4} \frac{\partial_{r} \Phi_{2}^{(2)}}{\partial \psi^{n}}-\frac{1}{2} \frac{\partial_{r} \Phi_{1}^{(2)}}{\partial \psi^{n}} \chi
$$

Ao aplicarmos a condição de consistência nos vínculos secundários não encontramos mais vínculos ou multiplicadores de Lagrange. Assim, $\lambda_{1}$ e $\lambda_{2}$ continuam indeterminados.

A Hamiltoniana modificada $H^{(1)}$, com o uso da nova forma do multiplicador de Lagrange $\lambda_{3, n}$ é escrita como

$$
\begin{aligned}
H^{(1)} & =i \chi \Phi_{1}^{(2)}-\frac{e}{2} \Phi_{2}^{(2)}+\lambda_{1} \Phi_{1}^{(1)}+\lambda_{2} \Phi_{2}^{(1)}+\left(-\frac{1}{2} \frac{\partial_{r} \Phi_{1}^{(2)}}{\partial \psi^{n}} \chi-\frac{e i}{4} \frac{\partial_{r} \Phi_{2}^{(2)}}{\partial \psi^{n}}\right) \Phi_{3, n}^{(1)} \\
& =-\frac{e}{2}\left(\Phi_{2}^{(2)}+\frac{i}{2} \frac{\partial_{r} \Phi_{2}^{(2)}}{\partial \psi^{n}} \Phi_{3, n}^{(1)}\right)-i\left(\Phi_{1}^{(2)}+\frac{i}{2} \frac{\partial_{r} \Phi_{1}^{(2)}}{\partial \psi^{n}} \Phi_{3, n}^{(1)}\right) \chi+\lambda_{1} \Phi_{1}^{(1)}+\lambda_{2} \Phi_{2}^{(1)}
\end{aligned}
$$

Usando

$$
T_{1}=\left(\Phi_{1}^{(2)}+\frac{i}{2} \frac{\partial_{r} \Phi_{1}^{(2)}}{\partial \psi^{n}} \Phi_{3, n}^{(1)}\right) \quad \text { e } \quad T_{2}=\left(\Phi_{2}^{(2)}+\frac{i}{2} \frac{\partial_{r} \Phi_{2}^{(2)}}{\partial \psi^{n}} \Phi_{3, n}^{(1)}\right)
$$


ou , de forma mais compacta,

$$
T=\Phi^{(2)}+\frac{i}{2}{\frac{\partial_{r} \Phi^{(2)}}{\partial \psi^{n}}}^{(1)} \Phi_{3 n},
$$

a Hamiltoniana modificada se torna

$$
H^{(1)}=-\frac{e}{2} T_{2}-i T_{1} \chi+\lambda_{1} \Phi_{1}^{(1)}+\lambda_{2} \Phi_{2}^{(1)}
$$

Com isso, o conjunto inicial de vínculos $\left(\Phi^{(1)}, \Phi^{(2)}\right)$ é equivalente ao conjunto de vín$\operatorname{culos}\left(\Phi^{(1)}, T\right)$.

Para continuarmos com a quantização é importante dividirmos os vínculos do sistema em vínculos de primeira classe e vínculos de segunda classe. Um vínculo é de primeira classe se o seu parêntese de Poisson, com todos os vínculos do sistema, é zero ou fracamente zero, ou seja, zero na superfície de vínculos. Se o vínculo possuir algum parêntese de Poisson diferente de zero, ele é um vínculo de segunda classe. $\mathrm{O}$ novo conjunto de vínculos pode ser dividido em um conjunto de vínculos de primeira classe, o conjunto $\left(\Phi_{1,2}^{(1)}, T\right)$, e em um conjunto de vínculos de segunda classe, $\Phi_{3 n}^{(1)}$.

\section{Fixação de calibre, parênteses de Dirac e quantização de Dirac}

Para seguir com a quantização do sistema, vamos fixar parcialmente o calibre da teoria impondo as condições de calibre suplementares $\Phi_{1,2}^{\mathrm{G}}=0$ aos vínculos primários $\Phi_{1,2}^{(1)}$

$$
\Phi_{1}^{\mathrm{G}}=\chi, \quad \Phi_{2}^{\mathrm{G}}=e-1 / m
$$

Para estes vínculos também devemos aplicar a condição de consistência $\dot{\Phi}_{1,2}^{\mathrm{G}}=$ $\left\{\Phi_{1,2}^{\mathrm{G}}, H^{(1)}\right\}=0$, que nos fornece os multiplicadores de Lagrange $\lambda_{1}=0$ e $\lambda_{2}=0$.

Com os novos vínculos, a classificação entre vínculos de primeira classe e de segunda classe mudou: $T$ é o conjunto de vínculos de primeira classe e $\varphi=\left(\Phi^{(1)}, \Phi^{\mathrm{G}}\right) \mathrm{o}$ e conjunto de vínculos de segunda classe.

O próximo passo para a quantização do sistema será usar o método de Dirac para o caso em que o sistema possui vínculos de primeira e segunda classes [61, 57]. Os passos que devemos seguir são os seguintes:

1. Calcular os parênteses de Dirac entre as variáveis do sistema. Eles fornecem as relações de comutação ou anticomutação entre os operadores do sistema. A relação de comutação ou anticomutação de dois operadores $\hat{\eta}$ e $\hat{\eta}^{\prime}$ do sistema quântico se relaciona com os parênteses de Dirac da seguinte forma:

$$
i\left\{\eta, \eta^{\prime}\right\}_{D(\Phi)}=\left[\hat{\eta}, \hat{\eta}^{\prime}\right]=\hat{\eta} \hat{\eta}^{\prime}-(-)^{P(\eta) P\left(\eta^{\prime}\right)} \hat{\eta}^{\prime} \hat{\eta} .
$$


O parêntese de Dirac de duas funções $F\left(q_{i}, p^{i}\right)$ e $G\left(q_{i}, p^{i}\right)$ na álgebra de Berezin é definido como

$$
\{F, G\}_{D(\Phi)}=\{F, G\}-\left\{F, \Phi_{l}\right\}\left(C^{l l^{\prime}}\right)^{-1}\left\{\Phi_{l^{\prime}}, G\right\},
$$

onde $\{F, G\}$ é o parêntese de Poisson na álgebra de Berezin das funções $F\left(q_{i}, p^{i}\right)$ e $G\left(q_{i}, p^{i}\right)$, e $\left(C^{l l^{\prime}}\right)^{-1}$ é o elemento $l l^{\prime}$ da matriz inversa da supermatriz cujos elementos $C^{l l^{\prime}}=\left\{\Phi_{l}, \Phi_{l^{\prime}}\right\}$ são os parênteses de Poisson entre todos os vínculos de segunda classe do sistema.

2. Os vínculos de segunda classe devem ter as suas variáveis substituídas pelos seus respectivos operadores e, em seguida, o vínculos devem ser igualados a zero:

$$
\varphi(\hat{\eta})=0
$$

onde $\varphi(\hat{\eta})$ são os os vínculos de segunda classe escritos em termos dos operadores $\hat{\eta}$. Estas são outras restrições, além das relações de comutação, que são impostas aos operadores $\hat{\eta}$.

3. Os vínculos de primeira classe também devem ter as suas variáveis substituídas pelos seus respectivos operadores. No entanto, eles não são iguais a zero, eles devem restringir os estados vetoriais:

$$
\xi(\hat{\eta})|\Psi\rangle=0,
$$

onde $\xi(\hat{\eta})$ são os os vínculos de primeira classe escritos em termos dos operadores $\hat{\eta}$.

Antes de começarmos a quantização do sistema, observamos que o conjunto de vínculos de segunda classe, $\varphi=\left(\Phi^{(1)}, \Phi^{G}\right)$, possui um subconjunto $\left(\Phi_{1,2}^{(1)}, \Phi^{G}\right)$ na chamada forma especial - para mais detalhes veja o capítulo 2 da referência [57] -, o que nos permite eliminar as variáveis $e, P_{e}, \chi, P_{\chi}$ da Hamiltoniana. Assim, as únicas variáveis que restam são $x, p, \psi^{n}$. Com isso, os parênteses de Dirac com respeito aos vínculos do conjunto $\varphi$ se reduzem aos parênteses de Dirac com respeito aos vínculos $\Phi_{3 n}^{(1)}$.

Podemos, então, iniciar o primeiro passo do procedimento descrito acima. O cálculo dos parênteses de Dirac nos fornece que

$$
\left\{x^{\alpha}, p_{\beta}\right\}_{D\left(\Phi_{3 n}^{(1)}\right)}=\delta_{\beta}^{\alpha}, \quad\left\{\psi^{n}, \psi^{m}\right\}_{D\left(\Phi_{3 n}^{(1)}\right)}=\frac{i}{2} \eta^{n m}
$$

Os outros parênteses de Dirac zeram. Com isso, as relações de comutação e anticomutação para os vínculos $\hat{x}, \hat{p}$ e $\hat{\psi}^{n}$, que correspondem respectivamente às variáveis $x, p$ e 
$\psi^{n}$, são:

$$
\begin{aligned}
& {\left[\hat{x}^{\alpha}, \hat{p}_{\beta}\right]_{-}=i\left\{x^{\alpha}, p_{\beta}\right\}_{D\left(\Phi_{3 n}^{(1)}\right)}=i \delta_{\beta}^{\alpha},} \\
& {\left[\hat{\psi}^{n}, \hat{\psi}^{m}\right]_{+}=i\left\{\psi^{n}, \psi^{m}\right\}_{D\left(\Phi_{3 n}^{(1)}\right)}=-\frac{1}{2} \eta^{n m} .}
\end{aligned}
$$

Para o segundo passo, escrevemos os vínculos de segunda classe em termos dos operadores do sistema:

$$
\hat{\Phi}_{3 n}^{(1)}=\hat{P}_{n}+i \hat{\psi}_{n}=0,
$$

onde as relações de comutação 4.25 e a equação 4.26 podem ser realizadas no espaço de 4-colunas $\Psi(x)$ que dependem de $x^{\alpha}$. Definimos que $\hat{x}^{\alpha}$ são os operadores de multiplicação pela variável $x^{\alpha}$ e que $\hat{p}_{\alpha}=-i \partial_{\alpha}, \hat{\psi}^{\alpha}=\frac{i}{2} \gamma^{5} \gamma^{\alpha}$, e $\hat{\psi}^{4}=\frac{i}{2} \gamma^{5}$, onde $\gamma^{n}$ são as matrizes de Dirac $\gamma,\left(\gamma^{\alpha}, \gamma^{5}\right)$, definidas no início deste capítulo.

Por fim, no último passo, devemos escrever os vínculos de primeira classe como operadores e aplicá-los nos vetores físicos. Lembramos que $T$ é o nosso conjunto de vínculos de primeira classe, vide eq. 4.23 . Como $\hat{\Phi}_{3 n}^{(1)}=0, \hat{T}$ se reduz aos operadores $\hat{\Phi}_{1}^{(2)}$ e $\hat{\Phi}_{2}^{(2)}$. Aplicando estes operadores em um estado físico, obtemos que

$$
\hat{\Phi}_{1,2}^{(2)} \Psi(x)=0 .
$$

Para a construção do operador $\hat{\Phi}_{1}^{(2)}$, realizamos as transformações

$$
x^{\alpha} \rightarrow x^{\alpha}, \quad p_{\alpha} \rightarrow-i \partial_{\alpha}, \quad \psi^{\alpha} \rightarrow \frac{1}{2} \gamma^{5} \gamma^{\alpha},
$$

o que nos fornece

$$
\begin{gathered}
\mathcal{P}_{\alpha}=-p_{\alpha}-\mathbb{A}_{\alpha}(x) \rightarrow i \partial_{\alpha}-\mathbb{A}(x)=\hat{\mathcal{P}}_{\alpha}, \\
d_{\alpha}=-2 i \epsilon_{\alpha \beta \mu \nu} K^{\beta}(x) \psi^{\mu} \psi^{\nu} \rightarrow \frac{i}{2} \epsilon_{\alpha \beta \mu \nu} K^{\beta}(x) \gamma^{\mu} \gamma^{\nu} .
\end{gathered}
$$

Assim escrevemos a função $\Phi_{1}^{(2)}$, da eq. 4.20$)$, como

$$
\Phi_{1}^{(2)}=\mathcal{P}_{\alpha} \psi^{\alpha}-m \psi^{4}+\frac{1}{3} \psi^{\alpha} d_{\alpha} \rightarrow \frac{i}{2} \gamma^{5}\left(\hat{\mathcal{P}}_{\alpha} \gamma^{\alpha}-m-\frac{i}{3 !} K^{\alpha}(x) \varepsilon_{\alpha \beta \mu \nu} \gamma^{\beta} \gamma^{\mu} \gamma^{\nu}\right)
$$

Substituindo o operador $\hat{\Phi}_{1}^{(2)}$ na primeira equação de 4.27 obtemos a equação de Dirac (4.4).

A construção do operador $\hat{\Phi}_{2}^{(2)}$ com o uso da função $\Phi_{2}^{(2)}$, eq. 4.21), gera um problema de ordenamento, já que o vínculo $\Phi_{2}^{(2)}$ possui termos que são o produto de momentos com funções das coordenadas. Para tais termos escolhemos a simetrização de Weyl para operadores, que nos fornece operadores hermitianos $\hat{\Phi}_{2}^{(2)}$. O principal motivo que nos levou a utilizar essa simetrização é que ela torna as equações em (4.27) 
consistentes. Com esse ordenamento obtemos que

$$
\hat{\Phi}_{2}^{(2)}=\left(\hat{\Phi}_{1}^{(2)}\right)^{2}
$$

ou seja, a segunda equação de 4.27 é consequência da primeira equação de 4.27). Ao aplicarmos o operador $\hat{\Phi}_{2}^{(2)}$ em um estado vetorial, seguindo a eq. 4.27), não obtemos novas equações. Dessa forma, as restrições que os vínculos de primeira classe colocam aos estados do sistema estão de acordo com o sistema inicialmente estudado, o que confirma a forma da ação obtida na seção anterior. 


\section{CAPÍTULO 5}

\section{Conclusão}

Dividimos este trabalho em três partes:

1. Revisão do trabalho de E. S. Fradkin e D. M. Gitman [1], com o objetivo de compreender o método de construção de modelos clássicos e pseudoclássicos ali desenvolvido.

2. Aplicação do método de Fradkin e Gitman ao sistema que descreve uma partícula fermiônica quiral que interage, através da força eletrofraca, com o campo de matéria e interage com o campo eletromagnético. Este é um resultado novo.

3. Quantização do modelo pseudoclássico obtido no item anterior, para mostrar explicitamente sua relação com a teoria quântica original e sua consistência.

Na primeira parte do trabalho, fizemos a revisão da referência [1], que desenvolve um método para a representação da função de Green causal através de integrais de trajetória para as equações de Klein-Gordon (partícula escalar) e de Dirac (partícula fermiônica). Ambas as equações foram acrescidas do termo que descreve a interação da partícula com o campo eletromagnético:

$$
\begin{aligned}
& {\left[\left(i \partial_{\mu}-g A_{\mu}(x)\right)^{2}-m^{2}\right] \Psi(x)=0,} \\
& {\left[\left(i \partial_{\mu}-g A_{\mu}(x)\right) \gamma^{\mu}-m\right] \Psi(x)=0 .}
\end{aligned}
$$

A partir dessas integrais de trajetória, pudemos extrair as correspondentes ações invariantes por transformação de calibre, e dessas ações pudemos extrair um modelo clássico para a partícula escalar e um modelo pseudoclássico para a partícula fermiônica, semelhantes ao modelo de Berezin-Marinov [3]. Esse último modelo é chamado pseudoclássico por conter variáveis de Grassmann associadas aos graus de liberdade de spin semi-inteiro.

Na segunda parte do trabalho aplicamos o método de Fradkin e Gitman em um sistema que está relacionado com o efeito magnético quiral. Este sistema é descrito 
pela equação de Dirac modificada para incluir a interação da partícula com o campo de matéria, através da interação eletrofraca, e com o campo eletromagnético externo:

$$
\left[\left(i \partial_{\mu}-g A_{\mu}(x)\right) \gamma^{\mu}-m-\gamma_{\mu}\left(V_{L}^{\mu}(x) P_{L}+V_{R}^{\mu}(x) P_{R}\right)\right] \Psi(x)=0
$$

Existe uma grande semelhança entre os passos realizados no sistema da eq.5.2 e os passos realizados neste sistema. A diferença entre estes passos está na maior complexidade das contas decorrente do termo de interação com o campo de matéria. Como nos casos anteriores, obtivemos, ao final do método, a representação da função de Green por integrais de trajetória e o modelo pseudoclássico do sistema.

Na terceira e última parte deste trabalho, utilizamos o modelo pseudoclássico da teoria descrita pela eq.5.3 para realizarmos a sua quantização seguindo a referência [57]. A Hamiltoniana e os vínculos da teoria foram obtidos pelo método de DiracBergmann. Através da fixação de calibre, foi possível encontrar um conjunto de vínculos de segunda classe. Com a aplicação da quantização de Dirac para o caso de sistemas com vínculos de primeira e segunda classe, obtivemos os parênteses de Dirac e as relações de comutação entre as variáveis do modelo. Essas relações de comutação permitiram encontrar a realização correta dos operadores correspondentes às variáveis. Esses operadores correspondentes às variáveis foram então usados para quantizar os vínculos de primeira classe, o que nos forneceu a equação de Dirac com que iniciamos o estudo deste sistema: a eq.5.3. 


\section{CAPÍTULO 6}

\section{Apêndice}

Neste apêndice provaremos que

$$
\begin{aligned}
T \exp \left[\left.\int_{0}^{1} \rho_{n}(\tau) \Gamma^{n} d \tau\right|_{\rho=0}\right] & =\exp \left[i \Gamma^{n} \frac{\partial_{l}}{\partial \theta^{n}}\right] \\
& \times\left.\int \exp \left[\int_{0}^{1}\left(\psi_{n} \dot{\psi}^{n}-2 i \rho_{n} \psi^{n}\right) d \tau+\psi_{n}(1) \psi^{n}(0)\right] D \psi\right|_{\theta=0} .
\end{aligned}
$$

Iniciamos com o uso do teorema de Wick na sua versão que nos diz que o ordenamento temporal de um funcional $F(\hat{\phi})$, sendo $\hat{\phi}(\tau)$ um conjunto de operadores, pode ser escrito como:

$$
T F(\hat{\varphi})=\left.\operatorname{Sym} \exp \left[\frac{1}{2} \int \frac{\delta r}{\delta \varphi\left(\tau_{1}\right)} K\left(\tau_{1}, \tau_{2}\right) \frac{\delta r}{\delta \varphi\left(\tau_{2}\right)} d \tau_{1} d \tau_{2}\right] F(\varphi)\right|_{\varphi=\hat{\varphi}},
$$

onde $K\left(\tau_{1}, \tau_{2}\right)=T \hat{\varphi}\left(\tau_{1}\right) \hat{\varphi}\left(\tau_{2}\right)-\operatorname{Sym} \hat{\varphi}\left(\tau_{1}\right) \hat{\varphi}\left(\tau_{2}\right)$. Para este caso usaremos $\hat{\varphi}(\tau)=\rho_{n}(\tau) \Gamma^{n}$ e $F(\hat{\phi})=\exp \int_{0}^{1} \hat{\phi}(\tau) d \tau$.

O ordenamento temporal $T$ satisfaz as condições:

$$
T\left\{\hat{\varphi}\left(\tau_{1}\right) \hat{\varphi}\left(\tau_{2}\right)\right\}= \begin{cases}\hat{\varphi}\left(\tau_{1}\right) \hat{\varphi}\left(\tau_{2}\right) & \text { se } \tau_{1}>\tau_{2}, \\ (-1)^{P\left(\hat{\phi}\left(\tau_{1}\right) P\left(\hat{\phi}\left(\tau_{2}\right)\right)\right.} \hat{\varphi}\left(\tau_{2}\right) \hat{\varphi}\left(\tau_{1}\right), & \text { se } \tau_{2}>\tau_{1},\end{cases}
$$

sendo $P(\hat{\phi}(\tau))$ a paridade do operador $\hat{\phi}(\tau)$.

Sym, o produto simetrizado, é calculado como

$$
\operatorname{Sym} \hat{\varphi}\left(\tau_{1}\right) \hat{\varphi}\left(\tau_{2}\right)=\frac{1}{2}\left[\hat{\varphi}\left(\tau_{1}\right) \hat{\varphi}\left(\tau_{2}\right)+(-)^{P\left(\hat{\phi}\left(\tau_{1}\right) P\left(\hat{\phi}\left(\tau_{2}\right)\right)\right.} \hat{\varphi}\left(\tau_{2}\right) \hat{\varphi}\left(\tau_{1}\right)\right] .
$$

Para esta prova usaremos que $\hat{\phi}(\tau)=\rho_{n}(\tau) \Gamma^{n}$, um operador par, já que $\rho_{n}(\tau)$ e $\Gamma^{n}$ 
são variáveis de Grassmann ímpares, o que nos fornece que

$$
\begin{aligned}
K\left(\tau_{1}, \tau_{2}\right) & =\hat{\varphi}\left(\tau_{1}\right) \hat{\varphi}\left(\tau_{2}\right)-\frac{1}{2}\left[\hat{\varphi}\left(\tau_{1}\right) \hat{\varphi}\left(\tau_{2}\right)+\hat{\varphi}\left(\tau_{2}\right) \hat{\varphi}\left(\tau_{1}\right)\right] \\
& =\frac{1}{2}\left[\hat{\varphi}\left(\tau_{1}\right) \hat{\varphi}\left(\tau_{2}\right)-\hat{\varphi}\left(\tau_{2}\right) \hat{\varphi}\left(\tau_{1}\right)\right], \text { se } \tau_{1}>\tau_{2}
\end{aligned}
$$

e

$$
K\left(\tau_{1}, \tau_{2}\right)=\frac{1}{2}\left[\hat{\varphi}\left(\tau_{2}\right) \hat{\varphi}\left(\tau_{1}\right)-\hat{\varphi}\left(\tau_{1}\right) \hat{\varphi}\left(\tau_{2}\right)\right], \text { se } \tau_{2}>\tau_{1} .
$$

Os resultados acima podem ser reescritos como

$$
K\left(\tau_{1}, \tau_{2}\right)=\frac{1}{2} \epsilon\left(\tau_{1}-\tau_{2}\right)\left[\hat{\varphi}\left(\tau_{1}\right), \hat{\varphi}\left(\tau_{2}\right)\right]_{-}, \operatorname{com} \epsilon(\tau)=\operatorname{sgn} \tau .
$$

Usando que $\hat{\varphi}(\tau)=\rho_{n}(\tau) \Gamma^{n}$, e que $\rho_{n}(\tau)$ anticomuta com $\Gamma^{n}$,

$$
K\left(\tau_{1}, \tau_{2}\right)=\frac{1}{2} \epsilon\left(\tau_{1}-\tau_{2}\right)\left[-2 \rho_{n}\left(\tau_{1}\right) \rho^{n}\left(\tau_{2}\right) \Gamma_{n} \Gamma^{n}\right] .
$$

A partir de $\left[\tilde{\gamma}^{m}, \tilde{\gamma}^{n}\right]_{+}=2 \eta^{m n}$, obtemos que

$$
K\left(\tau_{1}, \tau_{2}\right)=-\epsilon\left(\tau_{1}-\tau_{2}\right) \rho_{n}\left(\tau_{1}\right) \rho^{n}\left(\tau_{2}\right) .
$$

Voltando para a eq. 6.1) e fazendo a substituição $F(\hat{\phi})=\exp \int_{0}^{1} \hat{\phi}(\tau) d \tau$ obtemos que

$$
\left.\operatorname{Sym} \exp \left[\frac{1}{2} \int \frac{\delta_{r}}{\delta \varphi\left(\tau_{1}\right)} K\left(\tau_{1}, \tau_{2}\right) \frac{\delta_{r}}{\delta \varphi\left(\tau_{2}\right)} d \tau_{1} d \tau_{2}\right] \exp \left[\int_{0}^{1} \varphi(\tau) d \tau\right]\right|_{\varphi=\hat{\varphi}}
$$

A primeira exponencial pode ser expandida em série de Taylor. Com isso, a equação acima se torna

$$
\left.\sum_{n=0}^{\infty} \frac{1}{n !}\left(\frac{1}{2} \int \frac{\delta_{r}}{\delta \varphi\left(\tau_{1}\right)} K\left(\tau_{1}, \tau_{2}\right) \frac{\delta_{r}}{\delta \varphi\left(\tau_{2}\right)} d \tau_{1} d \tau_{2}\right)^{n} \exp \left[\int_{0}^{1} \varphi(\tau) d \tau\right]\right|_{\varphi=\hat{\varphi}} .
$$

O primeiro termo da equação acima, referente ao caso em que $n=0$, é $\left.\exp \left[\int_{0}^{1} \varphi(\tau) d \tau\right]\right|_{\varphi=\hat{\varphi}}$. O segundo termo, para $n=1$, é, a menos de uma constante, igual a

$$
\begin{aligned}
& \left(\frac{1}{2} \int \frac{\delta_{r}}{\delta \varphi\left(\tau_{1}\right)} K\left(\tau_{1}, \tau_{2}\right) \frac{\delta_{r}}{\delta \varphi\left(\tau_{2}\right)} d \tau_{1} d \tau_{2}\right) \exp \left[\int_{0}^{1} \varphi(\tau) d \tau\right] \\
& =\left(\frac{1}{2} \int \frac{\delta_{r}}{\delta \varphi\left(\tau_{1}\right)} K\left(\tau_{1}, \tau_{2}\right) \frac{\delta_{r}}{\delta \varphi\left(\tau_{2}\right)} \exp \left[\int_{0}^{1} \varphi(\tau) d \tau\right] d \tau_{1} d \tau_{2}\right),
\end{aligned}
$$


onde,

$$
\begin{aligned}
\frac{\delta_{r}}{\delta \varphi\left(\tau_{2}\right)} \exp \left[\int_{0}^{1} \varphi(\tau) d \tau\right] & =\exp \left[\int_{0}^{1} \varphi(\tau) d \tau\right] \int_{0}^{1} \frac{\delta_{r}}{\delta \varphi\left(\tau_{2}\right)} \varphi(\tau) d \tau \\
& =\exp \left[\int_{0}^{1} \varphi(\tau) d \tau\right] \int_{0}^{1} \delta\left(\tau-\tau_{2}\right) d \tau \\
& =\exp \left[\int_{0}^{1} \varphi(\tau) d \tau\right]
\end{aligned}
$$

O termo $\frac{\delta_{r}}{\delta \varphi\left(\tau_{1}\right)} \exp \left[\int_{0}^{1} \varphi(\tau) d \tau\right]$ é calculado da mesma forma. Temos, então, que

$$
\begin{aligned}
& \left(\frac{1}{2} \int \frac{\delta_{r}}{\delta \varphi\left(\tau_{1}\right)} K\left(\tau_{1}, \tau_{2}\right) \frac{\delta_{r}}{\delta \varphi\left(\tau_{2}\right)} d \tau_{1} d \tau_{2}\right) \exp \left[\int_{0}^{1} \varphi(\tau) d \tau\right] \\
& =\left(\frac{1}{2} \int K\left(\tau_{1}, \tau_{2}\right) d \tau_{1} d \tau_{2}\right) \exp \left[\int_{0}^{1} \varphi(\tau) d \tau\right]
\end{aligned}
$$

O terceiro termo da expansão, a menos de uma constante, é igual a

$$
\begin{aligned}
& \left(\frac{1}{2} \int \frac{\delta_{r}}{\delta \varphi\left(\tau_{1}\right)} K\left(\tau_{1}, \tau_{2}\right) \frac{\delta_{r}}{\delta \varphi\left(\tau_{2}\right)} d \tau_{1} d \tau_{2}\right)^{2} \exp \left[\int_{0}^{1} \varphi(\tau) d \tau\right] \\
& =\left(\frac{1}{2} \int \frac{\delta_{r}}{\delta \varphi\left(\tau_{1}\right)} K\left(\tau_{1}, \tau_{2}\right) \frac{\delta_{r}}{\delta \varphi\left(\tau_{2}\right)} d \tau_{1} d \tau_{2}\right)\left(\frac{1}{2} \int K\left(\tau_{1}, \tau_{2}\right) d \tau_{1} d \tau_{2}\right) \exp \left[\int_{0}^{1} \varphi(\tau) d \tau\right] \\
& =\left(\frac{1}{2} \int K\left(\tau_{1}, \tau_{2}\right) d \tau_{1} d \tau_{2}\right)\left(\frac{1}{2} \int \frac{\delta_{r}}{\delta \varphi\left(\tau_{1}\right)} K\left(\tau_{1}, \tau_{2}\right) \frac{\delta_{r}}{\delta \varphi\left(\tau_{2}\right)} d \tau_{1} d \tau_{2}\right) \exp \left[\int_{0}^{1} \varphi(\tau) d \tau\right] \\
& =\left(\frac{1}{2} \int K\left(\tau_{1}, \tau_{2}\right) d \tau_{1} d \tau_{2}\right)^{2} \exp \left[\int_{0}^{1} \varphi(\tau) d \tau\right] .
\end{aligned}
$$

É possível notar que

$$
\left.\sum_{n=0}^{\infty} \frac{1}{n !}\left(\frac{1}{2} \int \frac{\delta_{r}}{\delta \varphi\left(\tau_{1}\right)} K\left(\tau_{1}, \tau_{2}\right) \frac{\delta_{r}}{\delta \varphi\left(\tau_{2}\right)} d \tau_{1} d \tau_{2}\right)^{n} \exp \left[\int_{0}^{1} \varphi(\tau) d \tau\right]\right|_{\varphi=\hat{\varphi}}
$$

pode ser escrita como

$$
\begin{aligned}
& \left.\sum_{n=0}^{\infty} \frac{1}{n !}\left(\frac{1}{2} \int K\left(\tau_{1}, \tau_{2}\right) d \tau_{1} d \tau_{2}\right)^{n} \exp \left[\int_{0}^{1} \varphi(\tau) d \tau\right]\right|_{\varphi=\hat{\varphi}} \\
& =\exp \left[\frac{1}{2} \int K\left(\tau_{1}, \tau_{2}\right) d \tau_{1} d \tau_{2}\right] \exp \left[\int_{0}^{1} \varphi(\tau) d \tau\right] .
\end{aligned}
$$


Com isso, obtemos que

$$
\begin{aligned}
& \text { Symexp }\left.\left[\frac{1}{2} \int \frac{\delta_{r}}{\delta \varphi\left(\tau_{1}\right)} K\left(\tau_{1}, \tau_{2}\right) \frac{\delta_{r}}{\delta \varphi\left(\tau_{2}\right)} d \tau_{1} d \tau_{2}\right] \exp \left[\int_{0}^{1} \varphi(\tau) d \tau\right]\right|_{\varphi=\hat{\varphi}} \\
& =\exp \left[\frac{1}{2} \int K\left(\tau_{1}, \tau_{2}\right) d \tau_{1} d \tau_{2}\right] \exp \left[\int_{0}^{1} \varphi(\tau) d \tau\right]
\end{aligned}
$$

usando a eq. 6.2),

$$
\exp \left[\frac{1}{2} \int K\left(\tau_{1}, \tau_{2}\right) d \tau_{1} d \tau_{2}\right]=\exp \left[-\frac{1}{2} \iint_{0}^{1} \rho_{n}\left(\tau_{1}\right) \epsilon\left(\tau_{1}-\tau_{2}\right) \rho^{n}\left(\tau_{2}\right) d \tau_{1} d \tau_{2}\right]
$$

A seguir, utilizamos a equação (1.159) da referência [63]:

$$
\begin{aligned}
& \int D \xi \exp \left[\frac{1}{2}\left[\iint \xi_{n}\left(\tau_{1}\right) \mathcal{K}^{-1}\left(\tau_{1}, \tau_{2}\right) \xi^{n}\left(\tau_{2}\right) d \tau_{1} d \tau_{2}+\int \xi_{n}\left(\tau_{1}\right) A^{n}\left(\tau_{1}\right) d \tau_{1}\right]\right] \\
& =\epsilon \operatorname{det}\left(\frac{\mathcal{K}^{-1}}{2 \pi}\right)^{1 / 2} \exp \left(\frac{1}{2} \iint A_{n}\left(\tau_{1}\right) \mathcal{K}\left(\tau_{1}, \tau_{2}\right) A^{n}\left(\tau_{2}\right) d \tau_{1} d \tau_{2}\right)
\end{aligned}
$$

onde

$$
\epsilon \operatorname{det}\left(\frac{\mathcal{K}^{-1}}{2 \pi}\right)^{1 / 2}=\int D \xi \exp \left[\frac{1}{2} \iint \xi_{n}\left(\tau_{1}\right) \mathcal{K}^{-1}\left(\tau_{1}, \tau_{2}\right) \xi^{n}\left(\tau_{2}\right) d \tau_{1} d \tau_{2}\right] .
$$

$\xi^{n}(\tau)$, com $n=0, \ldots, 4$, são trajetórias ímpares.

Vemos que a última exponencial da eq. (6.4) pode ser escrita como

$$
\exp \left[-\frac{1}{2} \iint_{0}^{1} \rho_{n}\left(\tau_{1}\right) \epsilon\left(\tau_{1}-\tau_{2}\right) \rho^{n}\left(\tau_{2}\right) d \tau_{1} d \tau_{2}\right]
$$

se $A_{n}\left(\tau_{1}\right)=i \rho_{n}\left(\tau_{1}\right)$ e $\mathcal{K}\left(\tau_{1}, \tau_{2}\right)=\epsilon\left(\tau_{1}-\tau_{2}\right)$.

$\mathcal{K}\left(\tau_{1}, \tau_{2}\right)$ e $\mathcal{K}^{-1}\left(\tau_{1}, \tau_{2}\right)$ devem satisfazer a relação $\int \mathcal{K}^{-1}\left(\tau_{1}, \tau_{2}\right) \mathcal{K}\left(\tau_{1}, \tau_{2}\right) \xi\left(\tau_{2}\right) d \tau_{2}=\xi\left(\tau_{1}\right)$, esta relação é satisfeita se $\mathcal{K}^{-1}\left(\tau_{1}, \tau_{2}\right)=-\frac{1}{2} \frac{d}{d \tau_{2}}$, já que

$$
-\int \frac{1}{2} \frac{d}{d \tau_{2}} \epsilon\left(\tau_{1}-\tau_{2}\right) \xi\left(\tau_{2}\right) d \tau_{2}=\int \delta\left(\tau_{1}-\tau_{2}\right) \xi\left(\tau_{2}\right) d \tau_{2}=\xi\left(\tau_{1}\right),
$$

onde foi usado que $\frac{d}{d y} \epsilon(x-y)=-2 \delta(x-y)$. 
Utilizando essas substituições, vemos que

$$
\begin{aligned}
& \int D \xi \exp \left[-\frac{1}{2} \iint \xi_{n}\left(\tau_{1}\right) \frac{1}{2} \frac{d}{d \tau_{2}} \xi^{n}\left(\tau_{2}\right) d \tau_{1} d \tau_{2}\right] \\
& \times \exp \left[-\frac{1}{2} \iint_{0}^{1} \rho_{n}\left(\tau_{1}\right) \epsilon\left(\tau_{1}-\tau_{2}\right) \rho^{n}\left(\tau_{2}\right) d \tau_{1} d \tau_{2}\right] \\
& =\int D \xi \exp \left[\frac{1}{4}\left[-\iint \xi_{n}\left(\tau_{1}\right) \frac{d}{d \tau_{2}} \xi^{n}\left(\tau_{2}\right) d \tau_{1} d \tau_{2}+i \int \xi_{n}\left(\tau_{1}\right) \rho^{n}\left(\tau_{1}\right) d \tau_{1}\right]\right] .
\end{aligned}
$$

A exponencial $\exp \left[-\frac{1}{2} \iint_{0}^{1} \rho_{n}\left(\tau_{1}\right) \epsilon\left(\tau_{1}-\tau_{2}\right) \rho^{n}\left(\tau_{2}\right) d \tau_{1} d \tau_{2}\right]$ pode então ser escrita como

$$
\frac{\int D \xi \exp \left[-\iint_{0}^{1} \frac{1}{4} \xi_{n}\left(\tau_{1}\right) \dot{\xi}^{n}\left(\tau_{2}\right) d \tau_{1} d \tau_{2}+i \int \xi_{n}\left(\tau_{1}\right) \rho^{n}\left(\tau_{1}\right) d \tau_{1}\right]}{\int D \xi \exp \left[\frac{1}{4}\left[-\iint_{0}^{1} \xi_{n}\left(\tau_{1}\right) \dot{\xi}^{n}\left(\tau_{2}\right) d \tau_{1} d \tau_{2}\right]\right]}=\frac{J(\rho)}{J(0)},
$$

onde

$$
J(\rho)=\int D \xi \exp \left[-\iint_{0}^{1} \frac{1}{4} \xi_{n}\left(\tau_{1}\right) \dot{\xi}^{n}\left(\tau_{2}\right) d \tau_{1} d \tau_{2}+i \int \xi_{n}\left(\tau_{1}\right) \rho^{n}\left(\tau_{1}\right) d \tau_{1}\right] .
$$

Assim,

$$
J(0)=\int D \xi \exp \left[\frac{1}{4}\left[-\iint_{0}^{1} \xi_{n}\left(\tau_{1}\right) \dot{\xi}^{n}\left(\tau_{2}\right) d \tau_{1} d \tau_{2}\right]\right] .
$$

A próxima igualdade que será provada é:

$$
\exp \left[\int_{0}^{1} \rho_{n} \Gamma^{n} d \tau\right]=\exp \left[i \Gamma^{n} \frac{\partial_{l}}{\partial \theta^{n}}\right] \exp \left[-i \int_{0}^{1} \rho_{n}(\tau) \theta^{n} d \tau\right]
$$

Para isso, a exponencial $\exp \left[i \Gamma^{n} \frac{\partial_{l}}{\partial \theta^{n}}\right]$ é expandida em série de Taylor:

$$
\exp \left[i \Gamma^{n} \frac{\partial_{l}}{\partial \theta^{n}}\right] \exp \left[-i \int_{0}^{1} \rho_{n}(\tau) \theta^{n} d \tau\right]=\sum_{m=0}^{\infty} \frac{1}{m !}\left(i \Gamma^{n} \frac{\partial_{l}}{\partial \theta^{n}}\right)^{m} \exp \left[-\left.i \int_{0}^{1} \rho_{n}(\tau) \theta^{n} d \tau\right|_{\theta=0}\right]
$$

O primeiro termo do somatório acima, referente a $m=0$, é $\exp \left[-i \int_{0}^{1} \rho_{n}(\tau) \theta^{n} d \tau\right]$. O 
segundo termo, com $m=1$, é da forma

$$
\begin{aligned}
\left(i \Gamma^{n} \frac{\partial_{l}}{\partial \theta^{n}}\right) \exp \left[-i \int_{0}^{1} \rho_{n}(\tau) \theta^{n} d \tau\right] & =i \tilde{\gamma}^{n} \exp \left[-i \int_{0}^{1} \rho_{n}(\tau) \theta^{n} d \tau\right]\left[-i \int_{0}^{1} \frac{\partial_{l}}{\partial \theta^{n}}\left(\rho_{n}(\tau) \theta^{n}\right) d \tau\right] \\
& =i \tilde{\gamma}^{n} \exp \left[-i \int_{0}^{1} \rho_{n}(\tau) \theta^{n} d \tau\right]\left[i \int_{0}^{1} \frac{\partial_{l}}{\partial \theta^{n}}\left(\theta^{n} \rho_{n}(\tau)\right) d \tau\right] \\
& =i \tilde{\gamma}^{n} \exp \left[-i \int_{0}^{1} \rho_{n}(\tau) \theta^{n} d \tau\right]\left[i \int_{0}^{1} \rho_{n}(\tau) d \tau\right] \\
& =i \exp \left[-i \int_{0}^{1} \rho_{n}(\tau) \theta^{n} d \tau\right]\left[i \int_{0}^{1} \Gamma^{n} \rho_{n}(\tau) d \tau\right] \\
& =i \exp \left[-i \int_{0}^{1} \rho_{n}(\tau) \theta^{n} d \tau\right]\left[-i \int_{0}^{1} \rho_{n}(\tau) \Gamma^{n} d \tau\right] \\
& =\exp \left[-i \int_{0}^{1} \rho_{n}(\tau) \theta^{n} d \tau\right]\left[\int_{0}^{1} \rho_{n}(\tau) \Gamma^{n} d \tau\right] .
\end{aligned}
$$

Para o cálculo do terceiro termo é usado o resultado acima:

$$
\begin{aligned}
\left(i \Gamma^{n} \frac{\partial_{l}}{\partial \theta^{n}}\right)^{2} \exp \left[-i \int_{0}^{1} \rho_{n}(\tau) \theta^{n} d \tau\right] & =\left(i \Gamma^{n} \frac{\partial_{l}}{\partial \theta^{n}}\right) \exp \left[-i \int_{0}^{1} \rho_{n}(\tau) \theta^{n} d \tau\right]\left[\int_{0}^{1} \rho_{n}(\tau) \Gamma^{n} d \tau\right] \\
& =\exp \left[-i \int_{0}^{1} \rho_{n}(\tau) \theta^{n} d \tau\right]\left[\int_{0}^{1} \rho_{n}(\tau) \Gamma^{n} d \tau\right]^{2}
\end{aligned}
$$

Com isso, pode-se ver que os outros termos da expansão de Taylor também devem seguir o mesmo padrão dos termos acima:

$$
\begin{aligned}
& \sum_{m=0}^{\infty} \frac{1}{m !}\left(i \Gamma^{n} \frac{\partial_{l}}{\partial \theta^{n}}\right)^{m} \exp \left[-\left.i \int_{0}^{1} \rho_{n}(\tau) \theta^{n} d \tau\right|_{\theta=0}\right] \\
& =\sum_{m=0}^{\infty} \frac{1}{m !} \exp \left[-\left.i \int_{0}^{1} \rho_{n}(\tau) \theta^{n} d \tau\right|_{\theta=0}\right]\left[\int_{0}^{1} \rho_{n}(\tau) \Gamma^{n} d \tau\right]^{m} \\
& =\exp \left[-\left.i \int_{0}^{1} \rho_{n}(\tau) \theta^{n} d \tau\right|_{\theta=0}\right] \exp \left[\int_{0}^{1} \rho_{n}(\tau) \Gamma^{n} d \tau\right] \\
& =\exp \left[\int_{0}^{1} \rho_{n}(\tau) \Gamma^{n} d \tau\right] .
\end{aligned}
$$

Desse modo, a eq.6.5 está provada.

Pra finalizar, vamos organizar os resultados obtidos neste apêndice. Mostramos 
que

$T \exp \left[\left.\int_{0}^{1} \rho_{n}(\tau) \Gamma^{n} d \tau\right|_{\rho=0}\right]=\left.\operatorname{Sym} \exp \left[\frac{1}{2} \int \frac{\delta_{r}}{\delta \varphi\left(\tau_{1}\right)} K\left(\tau_{1}, \tau_{2}\right) \frac{\delta_{r}}{\delta \varphi\left(\tau_{2}\right)} d \tau_{1} d \tau_{2}\right] \exp \left[\int_{0}^{1} \varphi(\tau) d \tau\right]\right|_{\varphi=\hat{\varphi}}$ $=\exp \left[-\frac{1}{2} \iint_{0}^{1} \rho_{n}\left(\tau_{1}\right) \epsilon\left(\tau_{1}-\tau_{2}\right) \rho^{n}\left(\tau_{2}\right) d \tau_{1} d \tau_{2}\right] \exp \left[\int_{0}^{1} \varphi(\tau) d \tau\right]$

onde:

$$
\begin{gathered}
\varphi(\tau)=\rho_{n}(\tau) \tilde{\gamma}^{n} ; \\
\exp \left[-\frac{1}{2} \iint_{0}^{1} \rho_{n}\left(\tau_{1}\right) \epsilon\left(\tau_{1}-\tau_{2}\right) \rho^{n}\left(\tau_{2}\right) d \tau_{1} d \tau_{2}\right]=\frac{J(\rho)}{J(0)} ; \\
J(\rho)=\int D \xi \exp \left[-\iint_{0}^{1} \frac{1}{4} \xi_{n}\left(\tau_{1}\right) \dot{\xi}^{n}\left(\tau_{2}\right) d \tau_{1} d \tau_{2}+i \int \xi_{n}\left(\tau_{1}\right) \rho^{n}\left(\tau_{1}\right) d \tau_{1}\right] ; \\
\exp \left[\int_{0}^{1} \rho_{n}(\tau) \Gamma^{n} d \tau\right]=\left.\exp \left[i \Gamma^{n} \frac{\partial_{l}}{\partial \theta^{n}}\right] \exp \left[-i \int_{0}^{1} \rho_{n}(\tau) \theta^{n} d \tau\right]\right|_{\theta=0} .
\end{gathered}
$$

A junção das expressões acima, resultou em

$$
\begin{aligned}
& T \exp \left[\int_{0}^{1} \rho_{n}(\tau) \Gamma^{n} d \tau\right]=\frac{J(\rho)}{J(0)} \exp \left[i \Gamma^{n} \frac{\partial_{l}}{\partial \theta^{n}}\right] \exp \left[-\left.i \int_{0}^{1} \rho_{n}(\tau) \theta^{n} d \tau\right|_{\theta=0}\right] \\
= & \int D \xi \exp \left[-\iint_{0}^{1} \frac{1}{4} \xi_{n}\left(\tau_{1}\right) \dot{\xi}^{n}\left(\tau_{2}\right) d \tau_{1} d \tau_{2}+i \int \xi_{n}\left(\tau_{1}\right) \rho^{n}\left(\tau_{1}\right) d \tau_{1}\right] \\
& \times \exp \left[i \Gamma^{n} \frac{\partial_{l}}{\partial \theta^{n}}\right] \exp \left[-\left.i \int_{0}^{1} \rho_{n}(\tau) \theta^{n} d \tau\right|_{\theta=0}\right] .
\end{aligned}
$$

A seguir, foi feita a mudança de variáveis $\xi(\tau)+\theta=2 \psi(\tau)$ :

$$
\begin{aligned}
T \exp \left[\int_{0}^{1} \rho_{n}(\tau) \Gamma^{n} d \tau\right] & =\int D \psi \exp \left\{\left[\int_{0}^{1} \frac{1}{4}\left(4 \psi_{n} \dot{\psi}^{n}-2 \psi_{n} \dot{\theta}^{n}-2 \theta_{n} \dot{\psi} n+\theta_{n} \dot{\theta}^{n}\right)+\right.\right. \\
& \left.\left.-2 i \rho_{n} \psi^{n}+i \rho_{n} \theta^{n}\right] d \tau\right\} \exp \left[i \Gamma^{n} \frac{\partial_{l}}{\partial \theta^{n}}\right] \exp \left[-\left.i \int_{0}^{1} \rho_{n}(\tau) \theta^{n} d \tau\right|_{\theta=0}\right],
\end{aligned}
$$

onde $\dot{\theta}_{n}=0$, e o termo $-\frac{1}{2} \int_{0}^{1} \theta_{n} \dot{\psi}^{n} d \tau$ pode ser simplificado para

$$
-\frac{1}{2} \int_{0}^{1} \theta_{n} \dot{\psi}^{n} d \tau=-\frac{1}{2} \theta_{n}\left[\psi^{n}(1)-\psi^{n}(0)\right]
$$

Usando que $\xi(0)=-\xi(1)$, obteve-se que $\theta=\psi(1)+\psi(0)$. Como $\psi(0) \psi(0)=\psi(1) \psi(1)=0$, o resultado da integração acima se torna $\psi_{n}(1) \psi^{n}(0)$. Com isto, foi provado que 


$$
\begin{aligned}
T \exp \left[\left.\int_{0}^{1} \rho_{n}(\tau) \Gamma^{n} d \tau\right|_{\rho=0}\right] & =\exp \left[i \Gamma^{n} \frac{\partial_{l}}{\partial \theta^{n}}\right] \\
& \times\left.\int \exp \left[\int_{0}^{1}\left(\psi_{n} \dot{\psi}^{n}-2 i \rho_{n} \psi^{n}\right) d \tau+\psi_{n}(1) \psi^{n}(0)\right] D \psi\right|_{\theta=0}
\end{aligned}
$$




\section{Referências Bibliográficas}

[1] E.S.Fradkin, D.M.Gitman, Path integral representation for the relativistic particle propagators and BFV quantization, Phys. Rev. D 44 (1991) 3230-3236

[2] F.A.Berezin and M.S.Marinov, Pisma Zh. Eksp. Theor. Fiz. 21 (1975) 678, [JETP Lett. 21 (1975) 320]

[3] F.A.Berezin and M.S.Marinov, Ann. Phys. (N.Y.) 104 (1977) 336

[4] R.Casalbuoni, Nuovo Cimento A 33 (1976) 115; A.Barducci, R.Casalbuoni, and L.Lusanna, Nuovo Cimento A 35 (1976) 377

[5] L.Brink, S.Deser, B.Zumino, P.diVechia, and P.Howe, Phys. Lett. B 64 (1976) 435

[6] L.Brink, P.diVechia, and P.Howe, Nucl. Phys. B 118 (1977) 76

[7] M.Henneaux and C.Teteilboim, Ann. Phys. (N.Y.) 143 (1982) 127

[8] K.Sundermeyer, Constrained Dynamics, Lect. Notes Phys. Vol. 69 (Springer, Berlin 1982)

[9] P.D.Mannheim, Phys. Rev. D 32 (1985) 898; Phys. Lett. B 166 (1986) 191

[10] D.M.Gitman, I.V.Tyutin, Classical and quantum mechanics of the relativistic particle, Class. Quantum Grav. 7 (1990) 2131-2144

[11] S.P. Gavrilov, D.M. Gitman, Quantization of Point-Like Particles and Consisitent Relativistic Quantum Mechanics, Int. J. Mod. Phys. A15 (2000) 4499-4538; Quantization of the Relativistic Particle, Class.Quant.Grav. 17 issue 19 (2000) L133-L139; Quantization of a spinning particle in an arbitrary background, Class.Quant.Grav. 18 (2001) 2989-2998

[12] D.M.Gitman, A.V.Saa, Pseudoclassical Model of Spinning Particle with Anomalous Magnetic Momentum, Mod. Phys. Lett. A, 8 (1993) 463-468; Quantization of Spinning Particle with Anomalous Magnetic Momentum, Class. Quantum Grav. 10 (1993) 1447-1460. 
[13] D. Gitman and V.G. Kupriyanov, Path integral representations in noncommutative quantum mechanics and noncommutative version of Berezin-Marinov action, Europ. Phys. J. C 54 (2008) 325-332

[14] D.M. Gitman, A.E. Gonçalves and I.V. Tyutin, New pseudoclassical model for Weyl particles, Phys. Rev. D 50 (1994) 5439-5442; D.M. Gitman, A.E. Gonçalves and I.V. Tyutin, Quantization of a pseudoclassical model of the spin 1 relativistic particle, Int.J.Mod.Phys. A 10 (1995) 701-718.

[15] D.M. Gitman, A.E. Gonçalves and I.V. Tyutin, Pseudoclassical Supergauge Model for 2+1 Dirac Particle, Physics of Atomic Nuclei, 60 No.4 (1997) 748-752

[16] D.M. Gitman and A.E. Gonçalves, Pseudoclassical model for Weyl particle in 10 dimensions, J. Math. Phys. 38 (5) (1997) 2167-2170; Pseudoclassical description of the massive Dirac particles in odd dimensions, Int. J. Theor.Phys. 35 (1996) 2427-2438

[17] D.M. Gitman and Tyutin, Pseudoclassical model for Chern-Simons particles, Mod. Phys. Lett. A11 (1996) 381-388; Pseudoclassical description of higher spins in 2+1 dimensions, Int. J.Mod.Phys.A 12 (1997) 535-556

[18] D.M. Gitman, Path integrals and pseudoclassical description for spinning particles in arbitrary dimensions, Nucl. Phys. B 488 (1997) 490-512

[19] R. Fresneda, S. Gavrilov, D. Gitman, and P. Moshin, Quantization of $(2+1)$ spinning particles and bifermionic constraint problem, Class.Quant.Grav.21 (2004) 1419-1442

[20] B. Geyer, D.M. Gitman, and I. Shapiro, Path integral and pseudoclassical action for spinning particle in external electromagnetic and torsion fields, Int.J.Mod.Phys.A15 (2000) 3861-3876

[21] R. Fresneda and D. Gitman, Pseudoclassical description of scalar particle in nonAbelian background and path-integral representations, Intern. Journ. Mod. Phys. A 23 (6) (2008) 835-853.

[22] D.M. Gitman and S.I. Zlatev, Semiclassical Form of the Relativistic Particle Propagator, Mod. Phys. Lett.A 12 (1997) 2435-2443; Spin factor in path integral representation for Dirac propagator in external field, Phys. Rev. D55 (1997) 7701-7714

[23] D.M. Gitman, S.I. Zlatev and P.B. Barros, Application of Path Integration to Operator Calculus, J. Phys. A: Math.Gen. 31 (1998) 7791-7799

[24] C. Schubert, Perturbative Quantum Field Theory in the String-Inspired Formalism, Phys.Rept. 355 (2001) 73-234 
[25] Lyakhovich, S. L., A. Yu Segal, and A. A. Sharapov. Universal model of a $D=4$ spinning particle. Phys. Rev. D 54 (8) (1996): 5223.

[26] Son, Dam Thanh, and Naoki Yamamoto, Kinetic theory with Berry curvature from quantum field theories, Physical review D87 (2013): 085016.

[27] A. Vilenkin, Equilibrium parity-violating current in a magnetic field, Phys. Rev. D 22 (12), 3080 (1980).

[28] A. Vilenkin, Phys. Lett. B 80, 150 (1978).

[29] A. Vilenkin, Phys. Rev. D 20, 1807 (1979).

[30] G.M. Eliashberg, Zh. Eksp. Teor. Fiz. 38, 188 (1983).

[31] L.S. Levitov, Yu.V. Nazarov, and G.M. Eliashberg, Zh. Eksp. Teor. Fiz. 88, 229 (1985).

[32] A. N. Redlich and L. C. R. Wijewardhana, Phys. Rev. Lett. 54, 970 (1985).

[33] K. Tsokos, Phys. Lett. B 157, 413 (1985).

[34] C. G. Callan, Jr. and J. A. Harvey, Nucl. Phys. B 250, 427 (1985).

[35] B. Zumino, Y. -S. Wu and A. Zee, Nucl. Phys. B 239, 477 (1984).

[36] A.Yu. Alekseev, V.V. Cheianov, J. Fröhlich, Phys. Rev. Lett. 81, 3503 (1998).

[37] J. Frohlich and B. Pedrini, In ${ }^{\star}$ Fokas, A. (ed.) et al.: Mathematical physics $2000^{\star}$ 9-47 [hep-th/0002195].

[38] J. Frohlich and B. Pedrini, cond-mat/0201236.

[39] M. Joyce and M. E. Shaposhnikov, Phys. Rev. Lett. 79, 1193 (1997) [astro$\mathrm{ph} / 9703005]$.

[40] M. Giovannini and M. E. Shaposhnikov, Phys. Rev. Lett. 80, 22 (1998) [arXiv:hepph/9708303]; Phys. Rev. D 57, 2186 (1998) [arXiv:hepph/9710234].

[41] D. E. Kharzeev, The chiral magnetic effect and anomaly-induced transport, Progress in Particle and Nuclear Physics 75 (2014): 133-151.

[42] A.A. Abrikosov and S.D. Beneslavskiŭ, Sov. J. Exp. Theor. Phys. 32, 699 (1971); J. Low Temperature Phys. 5, 141 (1971).

[43] G. E. Volovik, Lect. Notes Phys. 718, 31 (2007). 
[44] X. Wan, A.M. Turner, A. Vishwanath and S.Y. Savrasov, Phys. Rev.B 83, 205101 (2011).

[45] A.A. Burkov and L. Balents, Phys. Rev. Lett. 95, 146802 (2011).

[46] A.A. Zyuzin, S. Wu, and A.A. Burkov, Phys. Rev. B 85, 165110 (2012); arXiv:1201.3624 [cond-mat.mes-hall].

[47] K. Kimura et al., Phys. Rev. B 78, 140401 (R) (2008).

[48] A. Rebhan, A. Schmitt, and S. A. Stricker, Anomalies and the Chiral Magnetic Effect in the Sakai-Sugimoto Model, J. High Energ. Phys. 2010, 26 (2010).

[49] Q. Li, D. E. Kharzeev, C. Zhang, Y. Huang, I. Pletikosić, A. V. Fedorov, R. D. Zhong, J. A. Schneeloch, G. D. Gu, and T. Valla, Chiral Magnetic Effect in ZrTe5, Nature Physics 12, (2016).

[50] G. Sigl, Astroparticle Physics: Theory and Phenomenology (Atlantis, Paris, 2017).

[51] H.B. Nielsen and M. Ninomiya, The Adler-Bell-Jackiw anomaly and Weyl fermions in a crystal, Phys. Lett. 130B,389 (1983).

[52] M.E. Peskin and D.V. Schroeder, An Introduction to Quantum Field Theory (Reading, Massachusetts, 1995)

[53] V. Koch, S. Schlichting, V. Skokov, P. Sorensen, J. Thomas, S. Voloshin, G. Wang, and H.-U. Yee, Status of the chiral magnetic effect and collisions of isobars, Chin. Phys. C 41, 072001 (2017).

[54] G. Sundaram and Q. Niu, Phys. Rev. B 59, 14915 (1999).

[55] Studenikin, A. I. (2008). Method of wave equations exact solutions in studies of neutrinos and electrons interaction in dense matter. Journal of Physics A: Mathematical and Theoretical, 41 (16), 164047.

[56] Dvornikov, M. (2018). Chiral magnetic effect in the presence of an external axialvector field. Physical Review D, 98(3), 036016.

[57] D. M .Gitman, I. V. Tyutin, Quantization of Fields with Constraints, (SpringerVerlag, Berlin 1990)

[58] J. Schwinger, On Gauge Invariance and Vacuum Polarization, Phys. Rev. 82, 664 (1951).

[59] F. A. Berezin and N. Mugibayashi, The Method of Second Quantization, 4. Dr. (Academic Press, San Diego, Calif., 1992). 
[60] M. Henneaux and C. Teitelboim. Quantization of gauge systems. 1992.

[61] P. A. M. Dirac, Lectures on Quantum Mechanics (Dover Publications, Mineola, NY, 2001).

[62] E. S. Fradkin. Some problems in functional formulation of quantum field theory. Conf. Proc., 89:1-34, 1967.

[63] A.N. Vasiliev. Functional Methods in Quantum Field Theory and Statistical Physics. Gordon \& Breach, 1997.

[64] R. P. Feynman, A. R. Hibbs, Quantum mechanics and path integrals (McGraw Hill, New York 1965)

[65] L.S. Schulman, Techniques and Applications of Path Integration (John Wiley and Sons 1981) 